Portland State University

PDXScholar

\title{
The Application of Exact Electrodiffusion Theory to Ion Transport Across Lipid Bilayer Membranes
}

\section{Scott Cohen}

Portland State University

Follow this and additional works at: https://pdxscholar.library.pdx.edu/open_access_etds

Part of the Atomic, Molecular and Optical Physics Commons

\section{Let us know how access to this document benefits you.}

\section{Recommended Citation}

Cohen, Scott, "The Application of Exact Electrodiffusion Theory to Ion Transport Across Lipid Bilayer Membranes" (1983). Dissertations and Theses. Paper 3245.

https://doi.org/10.15760/etd.3237

This Thesis is brought to you for free and open access. It has been accepted for inclusion in Dissertations and Theses by an authorized administrator of PDXScholar. Please contact us if we can make this document more accessible: pdxscholar@pdx.edu. 
AN ABSTRACT OF THE THESIS OF Scott Cohen for the Master of Science in Physics presented August $22,1983$.

Title: The Application of Exact Electrodiffusion Theory to Ion Transport Across Lipid Bilayer Membranes.

APPROVED BY MEMBERS OF THE THESIS COMMITTEE:

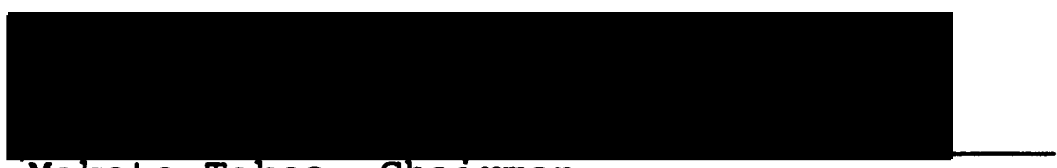

$$
\text { Makoto Takeo, Chairman }
$$

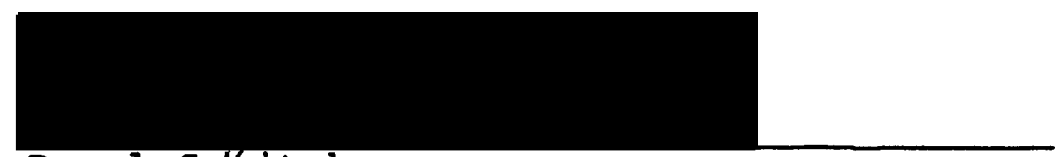

$$
\text { Pavel Smitek }
$$

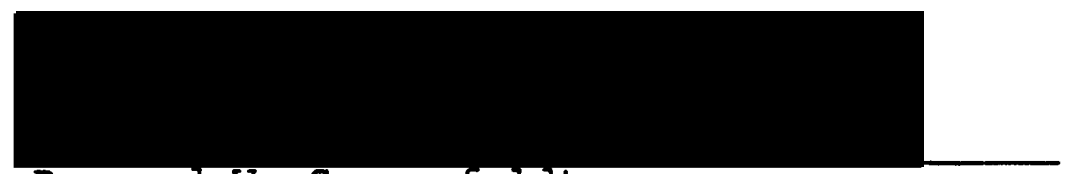

Raymond $\mathbf{w}$. Sommerfeldt

The question of how ions interact with each other and with the potential energy barrier in thin lipid bilayer membranes has interested investigators for several years. The application of electrodiffusion theory to the study of this question is the central theme of this work. We have calculated current-voltage curves for barriers of various shapes and heights, in each case by means of numerically integrating the exact electrodiffusion equation as well as 
this same equation in the constant field approximation. We have also calculated the total charge in the membrane for the same conditions under which we have calculated the current-voltage curves.

We present results which clearly indicate that both the height and the shape of the barrier are important in determining the current-voltage relation. They are also important in determining the total charge in the membrane, and in Appendix $C$, we suggest a possible method for measuring, experimentally, the amount of charge adsorbed in the membrane. Due to the presence of high charge densities near the interfaces of the membrane, the detailed shape of the barrier in this region appears to be of considerable importance in this regard.

The shape of the barrier is found also to be significant in determining the range of validity of the constant field approximation and this result is discussed in relationship to the important phenomenon of charge adsorption. 


\title{
THE APPLICATION OF EXACT ELECTRODIFFUSION \\ THEORY TO ION TRANSPORT ACROSS \\ LIPID BILAYER MEMBRANES
}

by

SCOTT COHEN

A thesis submitted in partial fulfillment of the requirements for the degree of

\author{
MASTER OF SCIENCE \\ in \\ PHYSICS
}

Portland State University 
TO THE OFFICE OF GRADUATE STUDIES AND RESEARCH:

The members of the Committee approve the thesis of Scott Cohen presented August 22, 1983.

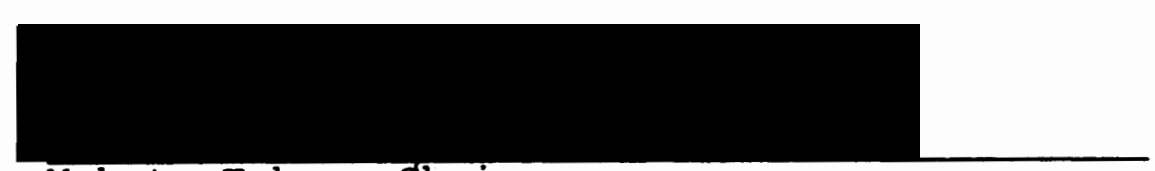

Makoto Takeo, Chairman

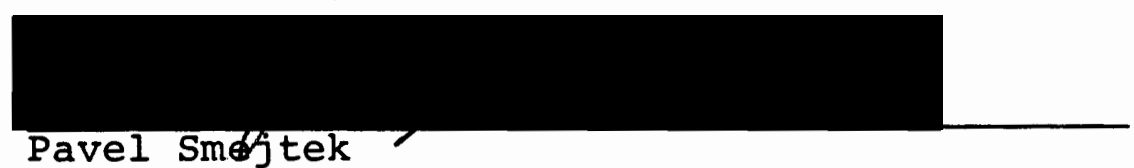

Pavel Smojtek

Raymond w. Sommerfeldt

APPROVED :

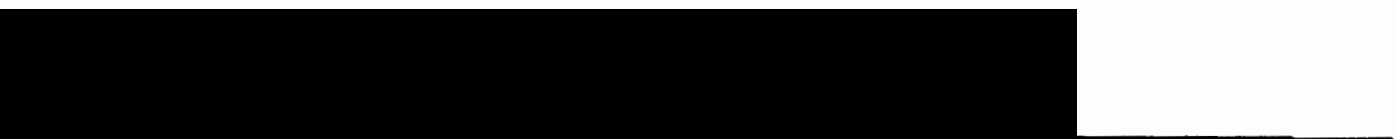

Raymond w. Sommerfeldt, Director, Physics

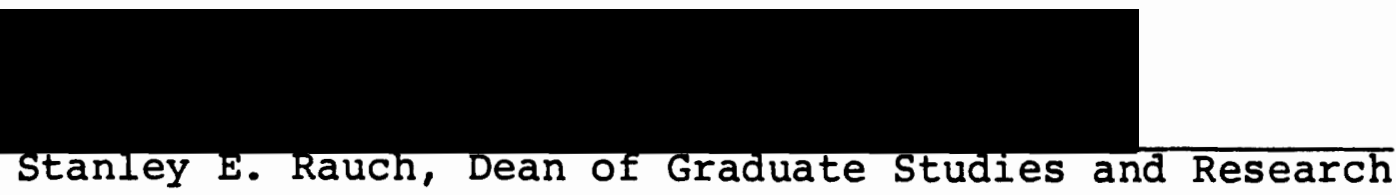


For my nieces,

Mahalia, Riva, Nichole, and Lalania.

Who have opened my eyes

to the wonder

- and wonderfulness -

of children. 


\section{ACKNOWLEDGEMENTS}

To Dr. Makoto Takeo, I wish to express my deep appreciation for his patience and generosity of spirit, and my great respect for his deep and comprehensive understanding of the principles of physics. He has always been ready and willing to sit and talk, even at times, to the extent, when deadlines have been pressing, of sacrificing previous commitments.

I also thank Dr. Mark Gurevitch for having made my introduction to graduate studies so much easier than it probably should have been. His sense of humor and refreshing perspectives on life have added something special to my years as a student at Portland State University.

Thanks also to all of my teachers for having shared, with myself and others, their insights and interests; and especially to Dr. Ray Sommerfeldt for his encouragement through the frustrating times; to Wes Brenner and Pankaj Shah of Academic Computing Services for their invaluable aid in my wanderings through that amazing world called computers; to Camille Hanson for always knowing what to do next, and how to do it; to Janet Eckelman for typing this manuscript; and to my colleagues, Bill Brown, David Lezak, and Robert McKay, for providing an atmosphere of friendship in which to work. 
I would also like to express my gratitude to all my good friends, and, especially, to Jackie clement for her constant warmth and understanding; and to all the free-flowing rivers of the Pacific Northwest for the peace of mind which they so willingly offer.

And, most of all, I would like to say thank you to my mother, my father, my sisters and their kids, for being something I will always be a part of - my family. 
TABLE OF CONTENTS

PAGE

DEDICATION. • • • • • • • • . . . . . . . . . iii ACKNOWLEDGEMENTS . . • . . . . . . . . . . . . iv LIST OF TABLES . • . • . . . . . . . . . . . . vii v vi LIST OF FIGURES . • . . . . . . . . . . . . . . viii CHAPTER

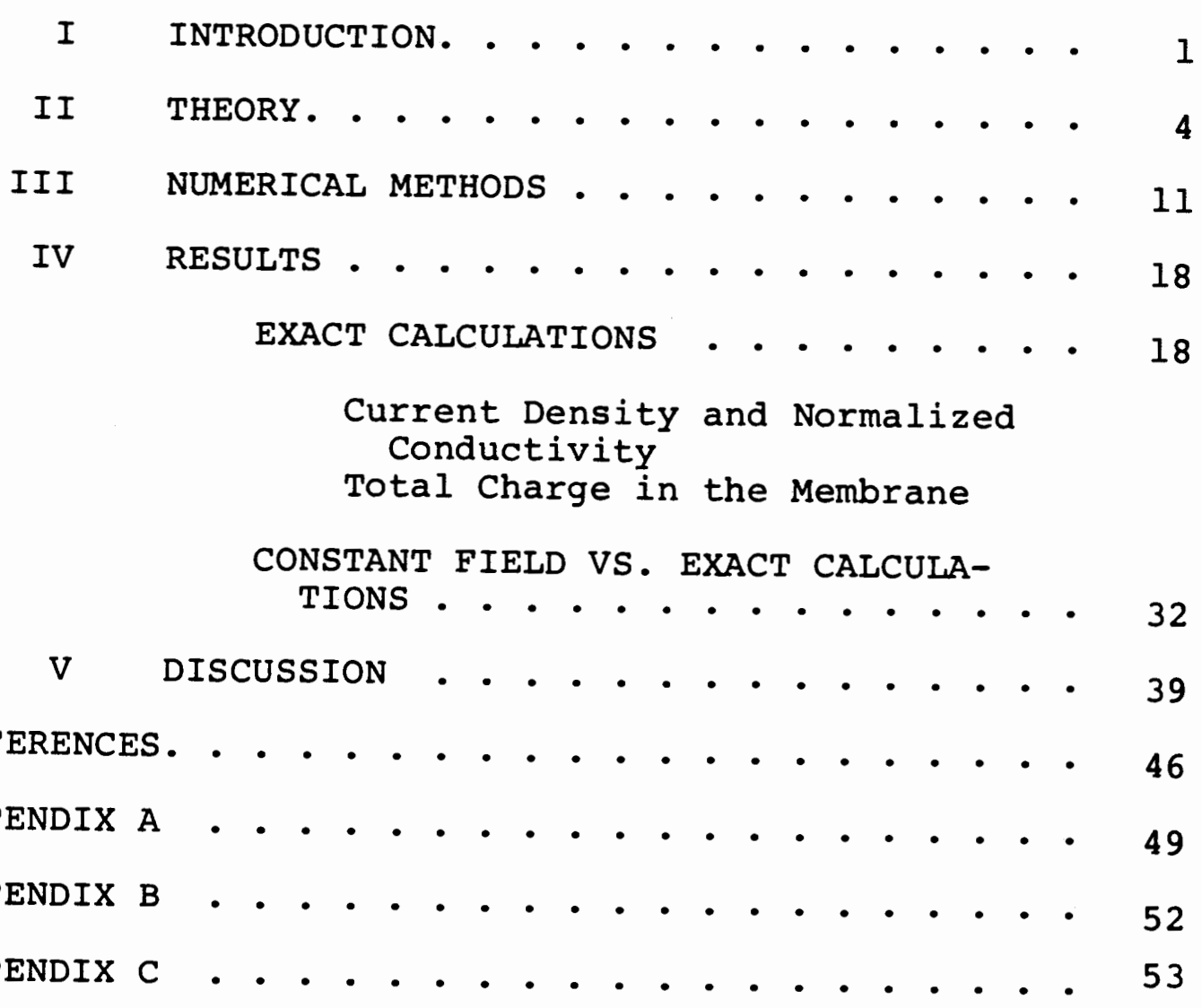




\section{LIST OF TABLES}

TABLE

PAGE

I Numerical Values of the Parameters Used in the Calculations. . . . . . . 16 
LIST OF FIGURES

FIGURE

PAGE

1. A Membrane Located Between Two Aqueous

Bathing Solutions, with a Depiction of

the Potential Energy Barrier. . . . .

2. Comparison of Current-Voltage Curves for

Various Peak Widths with a Peak Height of

$2.5 \mathrm{k}_{\mathrm{B}} \mathrm{T}$ and a Base Width of $1 / 3$ the

Membrane Width. . . . . . . . 20

3. Comparison of Conductivity-Voltage Curves for

the Same Barriers as in Figure 2. . . .

4. Comparison of Current-Voltage Curves for

Various Peak Widths with a Peak Height of

$5.0 \mathrm{k}_{\mathrm{B}} \mathrm{T}$ and a Base Width Equal to the

Membrane Width. . . . . . . . .

5. Comparison of Conductivity-Voltage Curves for

the Same Barriers as in Figure 4. . . .

6. Comparison of Current-Voltage Curves for

Various Peak Widths with a Peak Height

of $7.5 \mathrm{k}_{B} \mathrm{~T}$ and a Base Width Equal to the

Membrane Width. . . . . . . . .

7. Comparison of Conductivity-Voltage Curves for

the Same Barriers as in Figure 6. . . . 
F IGURE

PAGE

8. Comparison of Current-Voltage Curves for

Various Base Widths with a Peak Height of $2.5 \mathrm{k}_{B} \mathrm{~T}$ and a Peak Width of $7 / 30$ of the Membrane Width. . . . . . .

9. Comparison of Conductivity-Voltage Curves

for the Same Barriers as in Figure 8. .

10. Comparison of Current-Voltage Curves for various Base Widths with a Peak Height of $5.0 \mathrm{k}_{B} \mathrm{~T}$ and a Peak width of $7 / 30$ of the Membrane Width. . . . . . . .

11. Comparison of Conductivity-voltage Curves

for the Same Barriers as in Figure 10 .

12. Comparison of the Exact Calculations to those of the Constant Field Approximation with Current-Voltage Curves. .

13. Comparison of the Exact Calculations to those of the Constant Field Approximation with Conductivity-Voltage Curves . . . . . . . . . . .

14. Total Charge Adsorbed in the Membrane Plotted Against Aqueous Ion Concentration for Three Different Barriers. . 
15. Comparison of the Constant Field Approximation to the Exact Calculations Showing a Supralinear Relation in the Former, While the Latter Shows

a Superlinear Relation . . . . . . 38

16. Comparison of our Calculations to the Experimental Data of Stark and Benz (9). 44

17. Calculated Conductivity-Voltage Curves Showing a Transition from a Supralinear to a Superlinear Relation with Increasing Aqueous Ion Concentration. . 45 
CHAPTER I

INTRODUCTION

In the study of ion transport across thin lipid bilayer membranes, investigators have favored two approaches for the theoretical analysis. The first, the Eyring rate theory (1), assumes that ions move by means of a single jump from one side of the membrane to the other and between the membrane and the surrounding aqueous solution. The second, referred to as Nernst-Planck or electrodiffusion theory $(2,3,4)$, treats the membrane as a continuous medium through which the ions may move.

Each theory has its generalizations and approximations. For example, in the Eyring rate theory, the movement of ions across the membrane may be treated as a series of $\mathrm{n}$ discrete jumps (5). In electrodiffusion theory, the electric field as a function of position is important in determining the current through the membrane, and one may assume approximately that this field is constant $(6,7,8)$, whereas an exact treatment of the problem requires the introduction of the Poisson equation, which relates the variation of electric field to the charge density. When the charge density is small, this constant field approximation will yield results very close to those of 
the exact calculations.

Objections have occasionally been raised about the use of electrodiffusion theory in describing these systems. Stark and Benz (9) have contended that the large size of the diffusing ion - with diameters as much as one-fourth of the thickness of the membrane - may make this continuum theory inapplicable. They have also stated that electrodiffusion theory predicts only supralinear current - voltage relations, a statement which we will show in this thesis to be incorrect.

Yet, this theory has continued to be used and studied with some reasonable degree of success $(6,7,8)$. It is of interest to continue to study this theory, to look more closely at the physical properties which it predicts, and to investigate the conditions under which certain approximations, in particular that of a constant field, are valid. In fact, the standard form of this theory is itself an approximation when applied to thin membranes. Their thinness is important in contributing to their unique properties, since the electrostatic image forces (10) are significant over distances of the same order of magnitude as the membrane thickness (11). As a result, the potential energy of an ion in the membrane will vary strongly with position - being larger near the center by as much as 10 to $15 k_{B}{ }^{T}\left(k_{B^{\prime}}\right.$, the Boltzmann 
constant) at room temperature than near the interfaces (6) even if there is no applied potential and no other ions present in the membrane.

In the following, we refer to the potential energy profile in the membrane (not including applied voltage or ion-ion interactions) as the potential barrier. Several authors have discussed the form of this barrier $(10,11,12)$, and it is reasonably approximated by a symmetric trapezoid with a flat peak region in the center (6).

Standard electrodiffusion theory treats the potential profile as being constant, but we see that this may be a very unrealistic assumption. The effects of introducing the barrier directly into the equations of electrodiffusion theory have been studied $(6,7,8)$, but possibly because of the mathematical difficulties in treating the exact theory, investigators have always introduced the constant field approximation. In this paper, we study exact electrodiffusion theory in the steady state, with an emphasis upon looking at the effects of introducing different shaped barriers into the theoretical description of the membrane, and on comparing the exact calculations to those of the constant field approximation. 


\section{CHAPTER II}

\section{THEORY}

We consider the membrane to be a symmetric uniform dielectric slab located between two aqueous bathing solutions of high dielectric strength (see Figure 1). Ions present in the aqueous solution may penetrate into the membrane, either by themselves or by combining with a carrier molecule, such as valinomycin, at the membranesolution interface; but we assume for simplicity that only a single type of ion may penetrate in this way, so that no other ions will be present in the membrane.

The free current density will be a combination of diffusion due to concentration gradients and migration due to electrical forces (we assume that other types of forces do not act). This may be described by the Nernst-Planck or electrodiffusion equation (we use the ESU system of units throughout):

$$
j=-D\left(\frac{\partial \rho}{\partial x}-\frac{z F}{R T} E_{\rho}\right)
$$

with

$$
E=E_{\rho}+E_{b}-\frac{V}{d},
$$




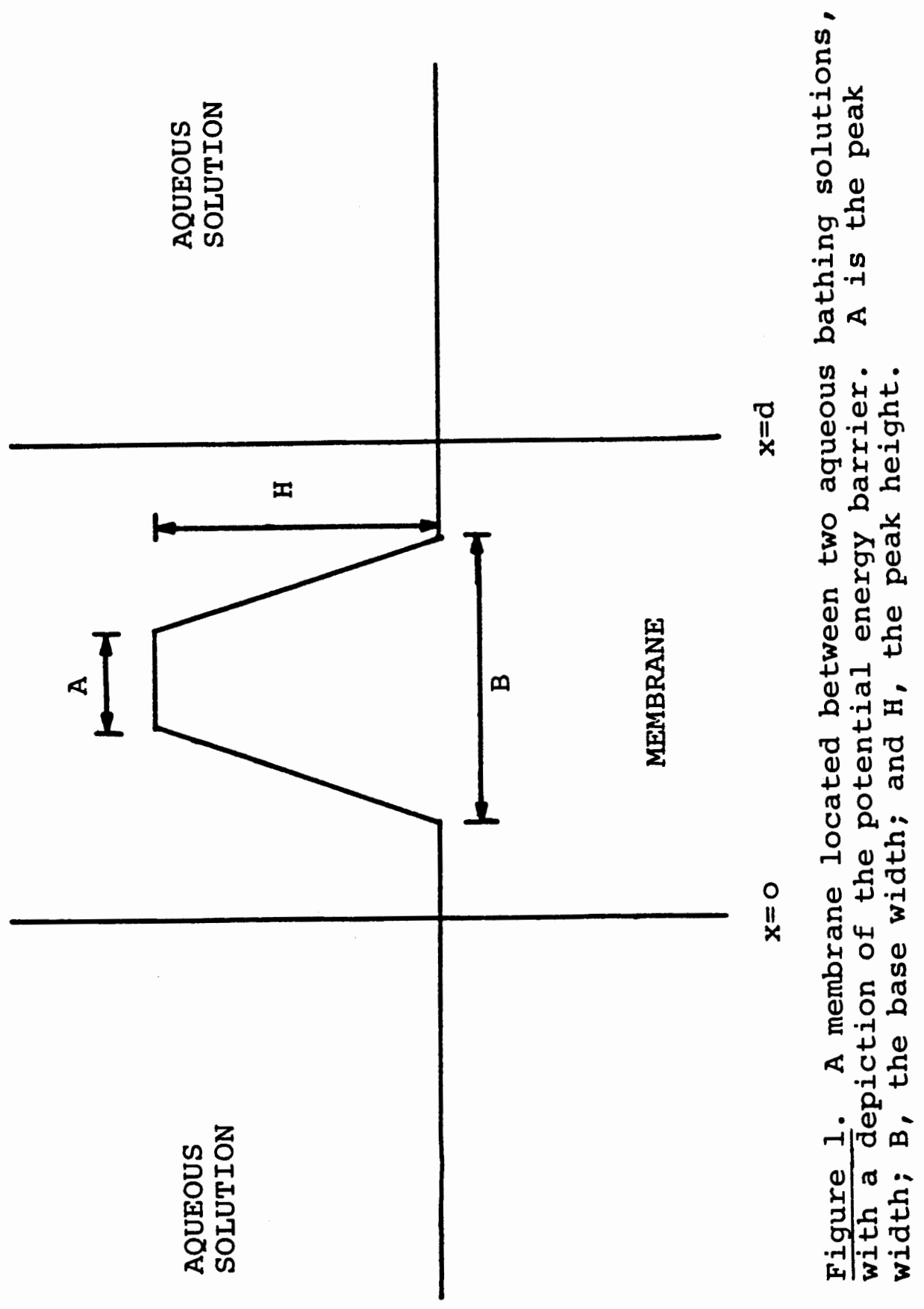


$E_{b}$ being the field due to the potential barrier; $V$, the voltage applied across the membrane; $\rho$, the charge density; and $E_{\rho}$, the electric field due to charges within the membrane and induced charges; and where $z$ is the valency of the ion; D, the diffusion coefficient; F, the Faraday constant; $\mathrm{R}$, the gas constant; and $\mathrm{T}$, the temperature. $E_{\rho}$ is related to the charge density through the Poisson equation,

$$
\frac{\partial E_{\rho}}{\partial x}=\frac{4 \pi}{\varepsilon} \rho
$$

where $\varepsilon$ is the membrane dielectric constant.

For time-dependent processes, we must also introduce the continuity equation,

$$
\frac{\partial j}{\partial x}+\frac{\partial D}{\partial t}=0
$$

which when combined with equation 1 , gives

$$
\frac{\partial \rho}{\partial t}-D \frac{\partial}{\partial x}\left(\frac{\partial \rho}{\partial x}-\frac{z F}{R T} E \rho\right)=0
$$

Integration with respect to $x$ and introduction of the Poisson equation gives an equation which describes the time-development of $E_{\rho}(x, t)$ and therefore $\rho(x, t)$ :

$$
-\frac{\partial E_{\rho}}{\partial t}-D\left(\frac{\partial^{2} E_{\rho}}{\partial x^{2}}-\frac{z F}{R T} E \frac{\partial E_{\rho}}{\partial x}\right)=\frac{4 \pi}{\varepsilon} J(t)
$$


Introduction of initial and boundary conditions will completely determine this system. $J(t)$ is the total current density - the sum of the free ionic current and the displacement current densities - which is observed in the external circuit (as free current only, since the displacement current is negligible there). Note that $J(t)$ is an integration constant and is, in this sense, not an independent variable.

In the steady state, the continuity equation tells us that the free ionic current is independent of position. Using this fact in equation 1 , one can multiply by the integrating factor,

$$
R(x)=e^{-\frac{z F}{R T} \int_{0}^{x} E\left(x^{\prime}\right) d x^{\prime}}
$$

to give

$$
\frac{d}{d x}[R(x) \rho(x)]=-\frac{j}{D} \quad R(x)
$$

Integrating over $\mathrm{x}$ from 0 to $d$, we obtain

$$
R(d) \rho(d)-\rho(0)=-\frac{j}{D} \int_{0}^{d} R(x) d x
$$

This may be rearranged to give the current density as

$$
j=D \frac{\rho(0)-\rho(d) e^{u}}{s_{0}^{d} e^{-\frac{z F}{R T}} \int_{0}^{x} E\left(x^{\prime}\right) d x^{\prime} d x}
$$


with $\mathrm{u}=\frac{\mathrm{zFV}}{\mathrm{RT}}$. The current density is also given by a direct integration, over $x$ from 0 to $d$, of equation 1 :

$$
j=\frac{D}{d}\left(\rho(0)-\rho(d)+\frac{z F}{R T} \int_{0}^{d} E(x) \rho(x) d x\right)
$$

Various relationships between important physical

parameters may be derived from equation 10 (see Appendix A).

Neumcke and Luuger (11) have used equation 10 to argue that ohmic behavior be expected for a membrane with a constant potential profile. Defining

$$
\begin{aligned}
& w(x)=-\frac{z F}{R T} \int_{0}^{x} E_{b}\left(x^{\prime}\right) d x^{\prime} \\
& \lambda(u)=j / u \\
& \lambda_{0}=\lim _{u \rightarrow 0} \lambda(u)=-\frac{D \rho(0)}{\int_{0}^{d} e^{w(x)} d x}
\end{aligned}
$$

and taking $E_{\rho}=0$ (constant field approximation), we have

$$
\frac{\lambda(u)}{\lambda_{0}}=-\frac{\rho_{\nu}(a)-\rho_{\nu}(d) e^{u}}{\rho_{0}(0) u} \cdot \frac{\int_{0}^{d} e^{w(x)} d x}{\int_{0}^{d} e^{w(x)+L x / d} d x} .
$$

$\rho_{\nu}(x)$ refers to the charge distribution at an applied voltage $V$, and $\rho_{\circ}(x)$ refers to the zero-voltage charge distribution. If $\rho_{\nu}(d)=\rho_{\nu}(0)=\rho_{0}(0)$, we have finally equation 19 of Neumcke and Lüuger (11):

$$
\frac{\lambda(u)}{\lambda_{0}}=\frac{e^{u}-1}{u} \cdot \frac{\int_{0}^{d} e^{w(x)} d x}{\int_{0}^{d} e^{w(x)+u x / d} d x} .
$$


This equation reduces to $\lambda(u)=\lambda_{0}$ for $w(x)=$ constant, describing ohmic behavior as stated. However, the assumption that the ion concentrations at the boundaries are equal and independent of applied voltage may not be appropriate in all cases. When this assumption does not hold, deviations from ohmic behavior, i.e. nonlinearities in the currentvoltage relation, must be expected. The nonlinearities, which are observed experimentally, will in part be due to the presence of the barrier, and in part, to the variation of the charge density between the two interfaces. We will show that for high charge densities - densities of the order of $10^{-13}$ to $10^{-12}$ moles $/ \mathrm{cm}^{2}$ will usually be sufficient - one may expect that ion-ion interactions will also play an important role.

It has also been shown that the zero-voltage conductivity, $\lambda_{0}$, when calculated by the constant field approximation, will always be greater than or equal to that when calculated exactly, if there is no barrier (13). Intuitively, one may expect that this is an effect of ion-ion interactions, their mutual repulsions making it more difficult for an ion to cross the membrane. However, it is unclear that the ion-ion interactions act in this way. The total charge in the membrane is determined from the numerical calculations, and it is seen to vary with barrier shape and 
applied voltage, as well as between the exact calculations and those of the constant field approximation. As will be shown, the mutual repulsions of the ions act to reduce the total charge in the membrane, which in turn lowers the conductivity. When we correct for this difference in total charge, so that it is the same in both calculations, then in some cases, the zero-voltage conductivity can be greater in the exact calculations than it is in the constant field approximation. The reduction of the total charge in the membrane due to ion-ion interactions plays an important role in determining the membrane conductivity. 


\section{CHAPTER III}

\section{NUMERICAL METHODS}

In the following, we deal with the differential form of the Nernst-Planck equation, rather than the integrated form. We divide the membrane into $\mathrm{n}$ small intervals and approximate 1 and 3 by difference equations, evaluating all relevant parameters at the midpoints of the divisions. To complete the problem, we must introduce boundary conditions. These we take to be the Eyring rate equations for transport across the interfaces $(14,15)$. Recalling that the current density is independent of position, we have

$$
\begin{array}{ll}
j=-k \sigma_{0}+F \beta k c, & x=0 \\
j=k \sigma_{d}-F \beta k c, & x=d
\end{array}
$$

where $k$ is the rate constant for ions jumping out of the membrane across the interface; $\beta$ is the partition coefficient, defined as $\beta=\sigma_{0} / F C$ for $V=0 ; c$, the concentration in the aqueous solution of the ions which move through the membrane; and $\sigma_{0, d}$, the surface charge density, inside the membrane, of these ions at the respective interface. 
Letting $r=4 \pi F d / \varepsilon R T$ and $h=1 / n$, we define nondimensional parameters as follows:

$$
\begin{array}{rlrl}
P_{i} & =r d \rho\left(\frac{d}{n} i\right) & e_{i}=\frac{F d}{R T} E\left(\frac{d}{n} i\right) \\
\gamma=\frac{r d}{D} j & \Sigma_{0, d}=r \sigma o \rho d \\
\delta=\frac{r d^{2}}{D} \beta k C & k=\frac{d^{2}}{D} k
\end{array}
$$

The subscript, $i$, labels the division.

We may now rewrite equations $1,3,17$, and 18 as

$$
\begin{aligned}
& \gamma=\frac{1}{4}\left(e_{i}+e_{i+1}\right)\left(P_{i}+P_{i+1}\right)-\left(P_{i+1}-P_{i}\right) / h \\
& P_{i}=\left(e_{i+1}^{0}-e_{i}^{0}\right) / h \\
& \gamma=-\left(k \Sigma_{0}-\delta\right) \\
& \gamma=k \Sigma_{d}-\delta
\end{aligned}
$$

and $e_{i}^{\rho}$ refers to the field due to charges in the membrane, defined analogously to $e_{i}$.

We take the surface charge density to be the average of the volume charge density in the first interval adjacent to the interface, multiplied by the width of the interval:

$$
\begin{aligned}
& \Sigma_{0}=\frac{1}{2} h\left(P_{0}+P_{1}\right) \\
& \Sigma_{d}=\frac{1}{2} h\left(P_{n-1}+P_{n}\right)
\end{aligned}
$$


Equation $17 \mathrm{a}$ is now used to eliminate the unknown current from equation $l a$, and inserting equation 20 for $\Sigma_{0}$ leaves a system of equations to determine the unknowns, $P_{i}$. If we now eliminate $P_{1}$ and $P_{n-1}$ from this system using equations 17a, 18a, 20 and 21 , we are left finally with a system of equations which can be solved easily by the method of Gaussian elimination:

$$
\begin{aligned}
& A_{1}^{\prime} P_{0}+B_{1} P_{2}=R_{0} \\
& R_{1} P_{0}+A_{i} P_{i}+B_{i} P_{i+1}=R_{2}, i=2, n-3 \\
& R_{1} P_{0}+A_{n-2} P_{n-2}+B_{n-2}^{\prime} P_{n}=R_{5} \\
& R_{1} P_{0}+R_{3} P_{n}=R_{3}+R_{4}
\end{aligned}
$$

with

$$
\begin{aligned}
& R_{0}=R_{2}-A_{1} \delta / R_{6} \\
& R_{1}=\kappa / R_{6} \\
& R_{2}=\delta\left(\frac{1}{h}-\frac{1}{4}\left(e_{0}+e_{1}\right)\right) / R_{6} \\
& R_{3}=\kappa / R_{7} \\
& R_{4}=\delta\left(\frac{1}{h}+\frac{1}{4}\left(e_{n-1}+e_{n}\right)\right) / R_{7} \\
& R_{5}=R_{2}-B_{n-2} \delta / R_{7}
\end{aligned}
$$




$$
\begin{aligned}
& R_{6}=\frac{1}{2} k h-\frac{1}{h}+\frac{1}{4}\left(e_{0}+e_{1}\right) \\
& R_{7}=\frac{1}{2} k h-\frac{1}{h}-\frac{1}{4}\left(e_{n-1}+e_{n}\right) \\
& A_{i}=-\left(\frac{1}{h}+\frac{1}{4}\left(e_{i}+e_{i+1}\right)\right), i=1, n-2 \\
& B_{i}=\frac{1}{h}-\frac{1}{4}\left(e_{i}+e_{i+1}\right), \quad i=1, n-2 \\
& A_{1}^{-}=R_{1}-\left(R_{6}+\frac{2}{h}\right) / R_{6} \\
& B_{n-2}^{\prime}=-B_{n-2}\left(R_{7}+\frac{2}{h}\right) / R_{7}
\end{aligned}
$$

The presence of the product term, $E(x) p(x)$ in equation 1 (or $e_{i} P_{i}$ in equation $\left.l a\right)$, and the fact that $E(x)$ depends upon $\rho(x)$ through equation 3 introduces difficulties into the solution of this nonlinear system of equations. We do not know $E(x)$ until we know $\rho(x)$ and vice-versa. Thus, it is necessary to utilize an iterative technique whereby an initial guess for the $e_{i}$ 's is made - we use the constant field approximation, here; $e_{i}=\nu=\frac{F V}{R T}$ - and the system of equations solved for the $\mathrm{P}_{i}$ 's. Then a new estimate of the $e_{i}$ 's is found from equation $3 a$, using these values of the $\mathrm{P}_{i}$ 's, and we begin again. This process is repeated until the $e_{i}$ 's are observed not to change with further iteration to within a convergence factor which is initially taken to be .001 . Then, from the known values of the $P_{i}$ 's and $e_{i}$ 's, the current can be found from the difference equation form of equation 10 , 


$$
\gamma=P_{0}-P_{n}+\frac{h}{4} \sum_{i=0}^{n-1}\left(e_{i}+e_{i+1}\right)\left(P_{i}+P_{i+1}\right)
$$

or from what turns out to be more accurate, equations 17a and 18a. If these give values for the current which differ by more than $5 \%$, the original convergence factor is divided by 10 and the iterative process is continued.

Unfortunately, under most conditions, this iterative process will not converge. It is necessary to force convergence, using an averaging technique (16), whereby we do not use the new $e_{i}$ 's directly, but average them with previously computed values and use the averaged result in the next computation.

More specifically, the next guess for the $e_{i}$ 's is calculated as (16)

$$
e_{i}(\text { new })=\left(1-\alpha_{i}\right) e_{i}^{0}+\alpha_{i}\left(\beta_{1} e_{i}^{1}+\beta_{2} e_{i}^{2}+\beta_{3} e_{i}^{3}\right)
$$

Here, $e_{i}^{0}$ is the previously used value of $e_{i}$ (new) $e_{i}^{1}$, the value of $e_{i}$ which was calculated from the $P_{i}$ 's on the most recent iteration; $e_{i}^{2}$, that calculated from the $P_{i}$ 's on the iteration before that; etc. We take $\beta_{1}=4 / 7, \beta_{2}=2 / 7, \beta_{3}=1 / 7$ (note that they are normalized, $\beta_{1}+\beta_{2}+\beta_{3}=1$ ), and the $\alpha_{i}$ vary through the computation as described by offner (16), with the initial values set equal to .1. In our calculations, convergence is usually achieved within 15 to 20 iterations, 
being, in general, slower for steeper barriers and/or higher concentrations. In fact, for very steep barriers, the method may not converge at all; either the $e_{i}$ 's become very large, or the $P_{i}$ 's are negative at the end of the calculation - by definition, they must be greater than or equal to zero - or the different values calculated for the current always differ by too large an amount.

Table I shows the numerical values of the parameters used in our calculations. Where relevant, all values have been taken from the results of Stark, et. al. (17) on the transport of the valinomycin $-\mathrm{K}^{+}$complex (see Appendix B).

TABLE I

NUMERICAL VALUES OF THE

PARAMETERS USED IN

THE CALCULATIONS

\begin{tabular}{llllll}
\hline$\varepsilon$ & $\mathrm{d}(\mathrm{nm})$ & $\mathrm{T}\left(\mathrm{O}_{\mathrm{K}}\right)$ & $\mathrm{k}\left(\mathrm{sec}^{-1}\right)$ & $\beta(\mathrm{cm})$ & $\mathrm{D}\left(\mathrm{cm}^{2} / \mathrm{sec}\right)$ \\
2.0 & 4.0 & 300 & $4.55 \times 10^{4}$ & $1.29 \times 10^{-9}$ & $1.46 \times 10^{-9}$ \\
\hline
\end{tabular}

The boundary conditions which we have used are appropriate to transport of hydrophobic ions $(14,15,18)$ but it is easily shown that if the uncomplexed carrier concentration is the same on each side of the membrane, then the boundary conditions for carrier-mediated transport reduce to the same 
form as those which we have used (see Appendix B). For the calculations, we use trapezoidal barriers, varying the base width (taking on values equal to $\frac{1}{3}, \frac{2}{3}$ and 1.0 of the membrane width), the peak width (taking on values equal to $1,7,13,19$, and 25 thirtieth's of the membrane width), and peak height (with values of $2.5,5.0,7.5$, and $10.0 \mathrm{k}_{\mathrm{R}^{\mathrm{T}}}$ ). Also, we vary the aqueous concentration of ions, $c$, to take on the values .01, $.05, .1, .5$, and 1.0 in moles/liter. 
CHAPTER IV

RESULTS

For the sake of clarity, we first list some of the gross features of the results of the calculations and discuss some specific examples.

\section{EXACT CALCULATIONS}

Current Density and Normalized Conductivity

(1) The current density, and hence conductivity, $\lambda(u)$, at any given voltage is always smaller for greater peak widths and peak heights in the given potential barrier.

(2) The normalized conductivity, $\lambda(u) / \lambda_{0}$, generally increases with increasing peak width.

(3) The variations with peak width are more pronounced the higher the barrier and the narrower the base width.

At a height of $2.5 \mathrm{k}_{B} \mathrm{~T}$, and full base width, the $\mathrm{j}-\mathrm{V}$ curves for different peak widths are virtually indistinguishable, though small differences are recognizable in the conductivity curves. For very narrow base widths, even at this small peak height, very large differences occur with 
only small variations in peak width, and these are especially noticeable in the conductivity curves (see Figures 2 and 3 ). 1

At heights of $5.0 \mathrm{k}_{3} \mathrm{~T}$ and $7.5 \mathrm{k}_{\mathrm{B}} \mathrm{T}$, large variations with peak width are apparent in both types of plots (see Figures 4-7), while at $10.0 \mathrm{k}_{\mathrm{B}} \mathrm{T}$, the normalized conductivity curves are beginning to coalesce, at least at low to moderate voltages, even though the current may differ by a factor of 3 to 4 at some voltages.

(4) The current density at any given voltage is always less for narrower base widths.

(5) The normalized conductivity always increases with decreasing base width.

(6) These variations with base width are more pronounced the higher the barrier and the greater the peak width (see Figures 8-11).

One may note that a variation in base width seems to have more of an effect on these curves than does a comparable variation in peak width (compare Figures 4 and 5 with Figures 10 and 11, noting that the solid line in the

\footnotetext{
${ }^{1}$ All plots labeled "conductivity" refer to normalized conductivity, $\lambda(u) / \lambda_{0}$.
} 


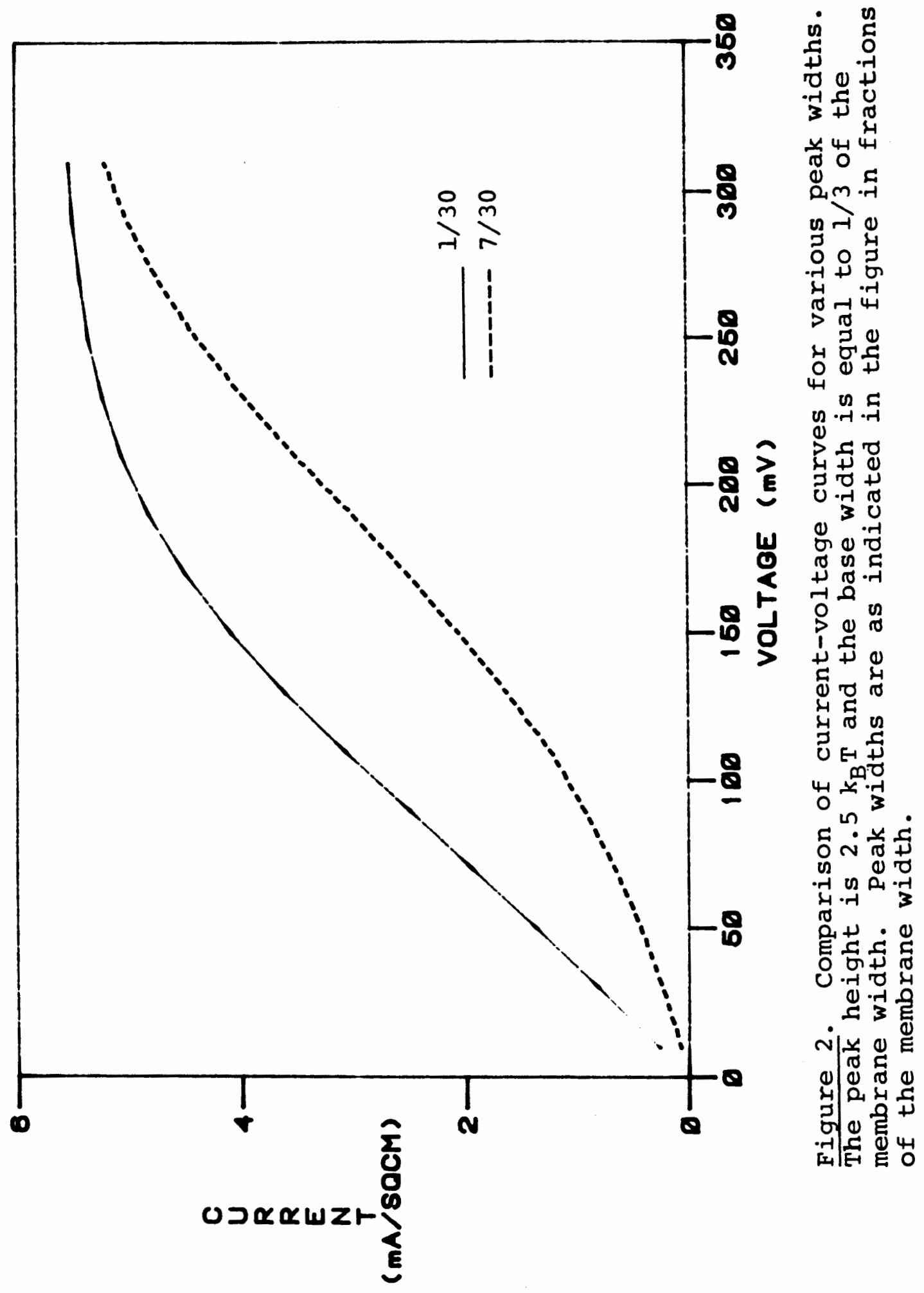




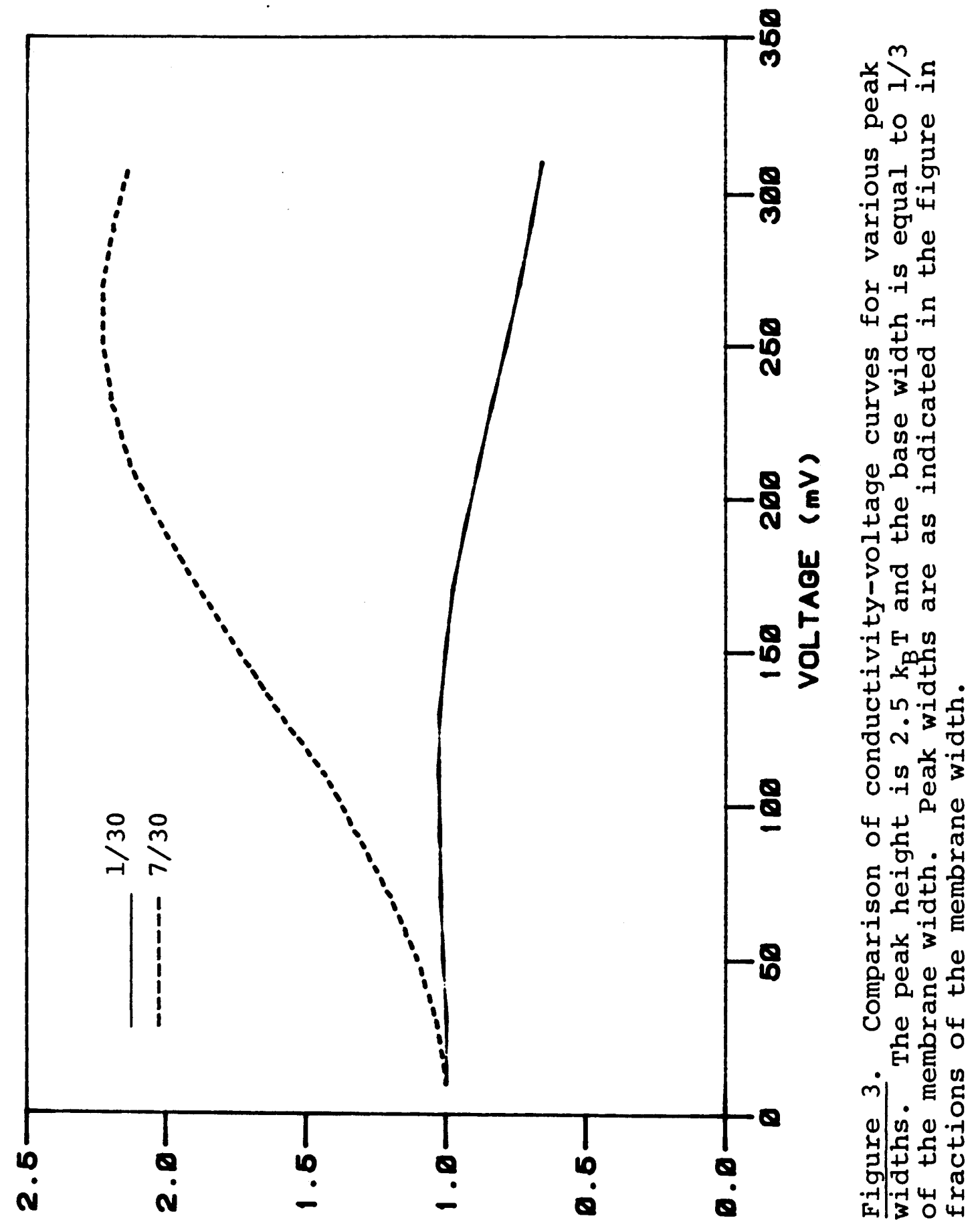




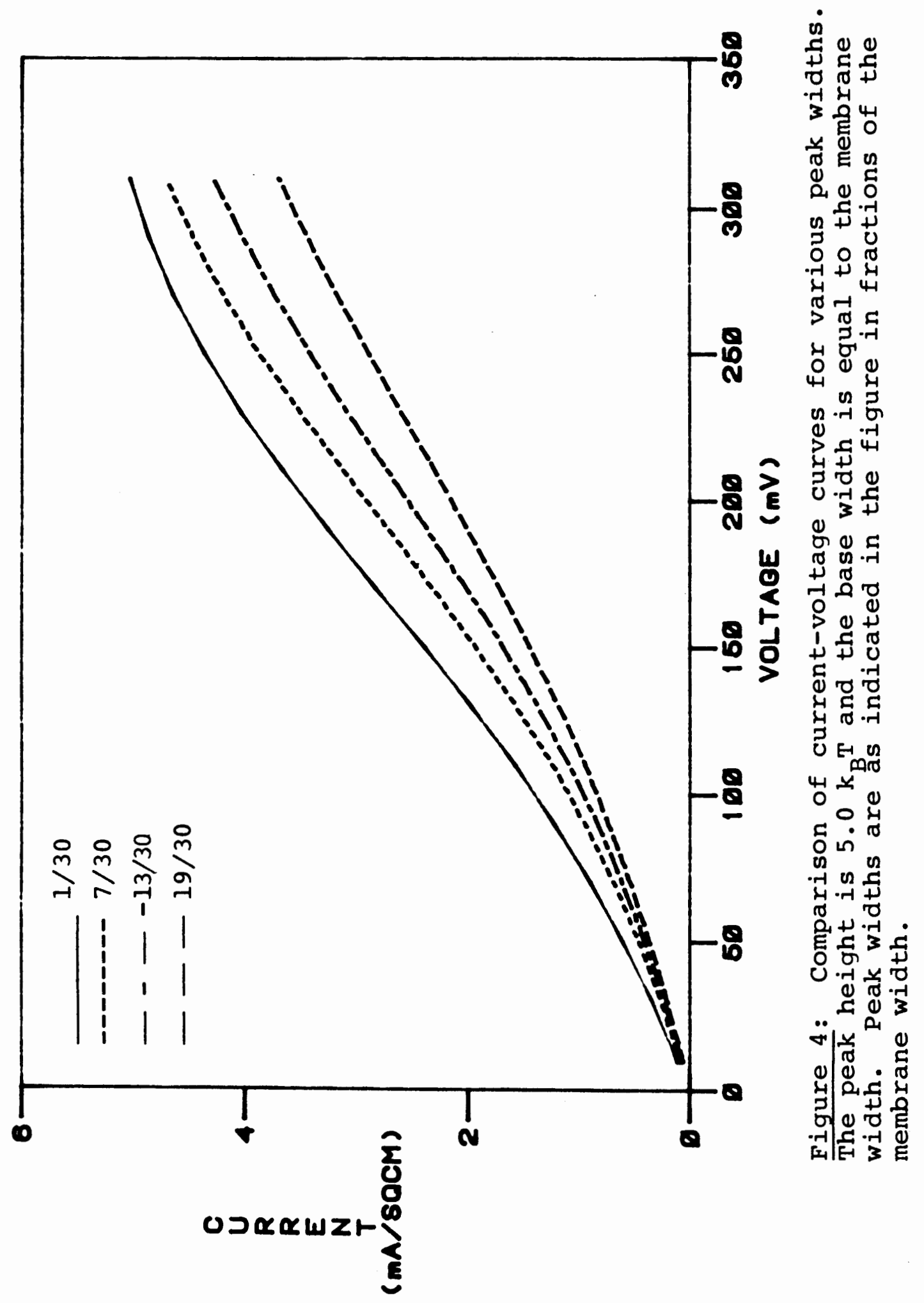




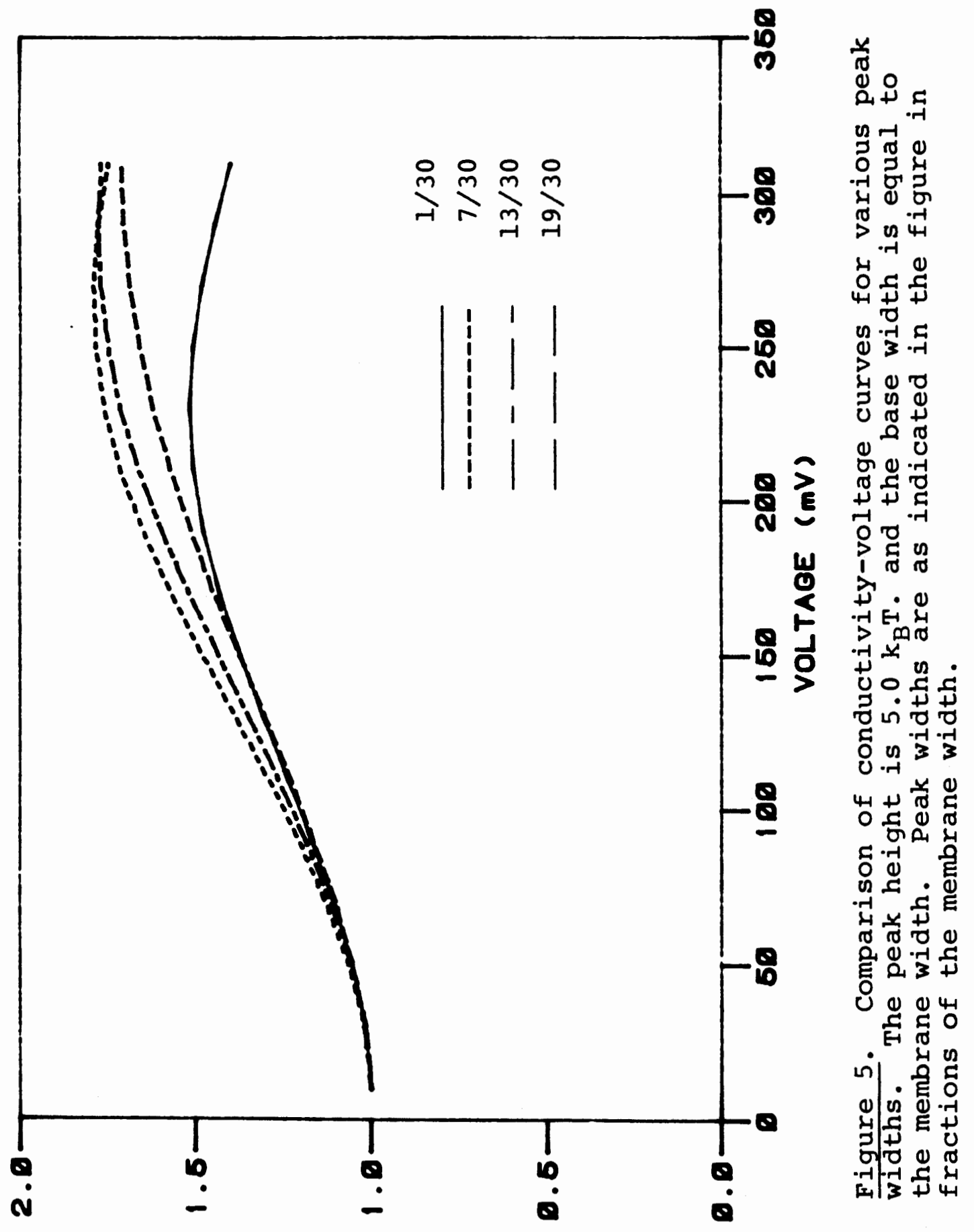




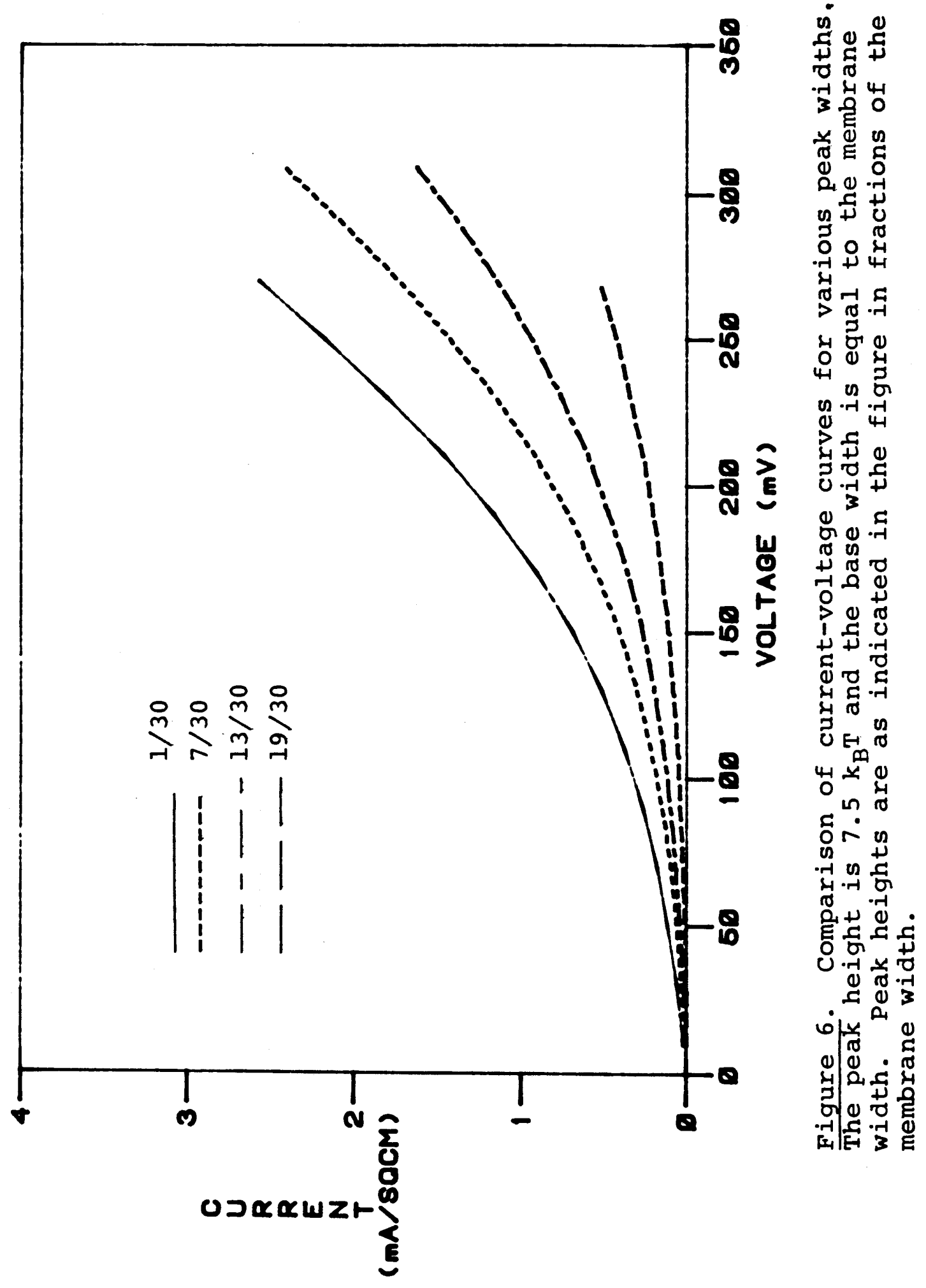




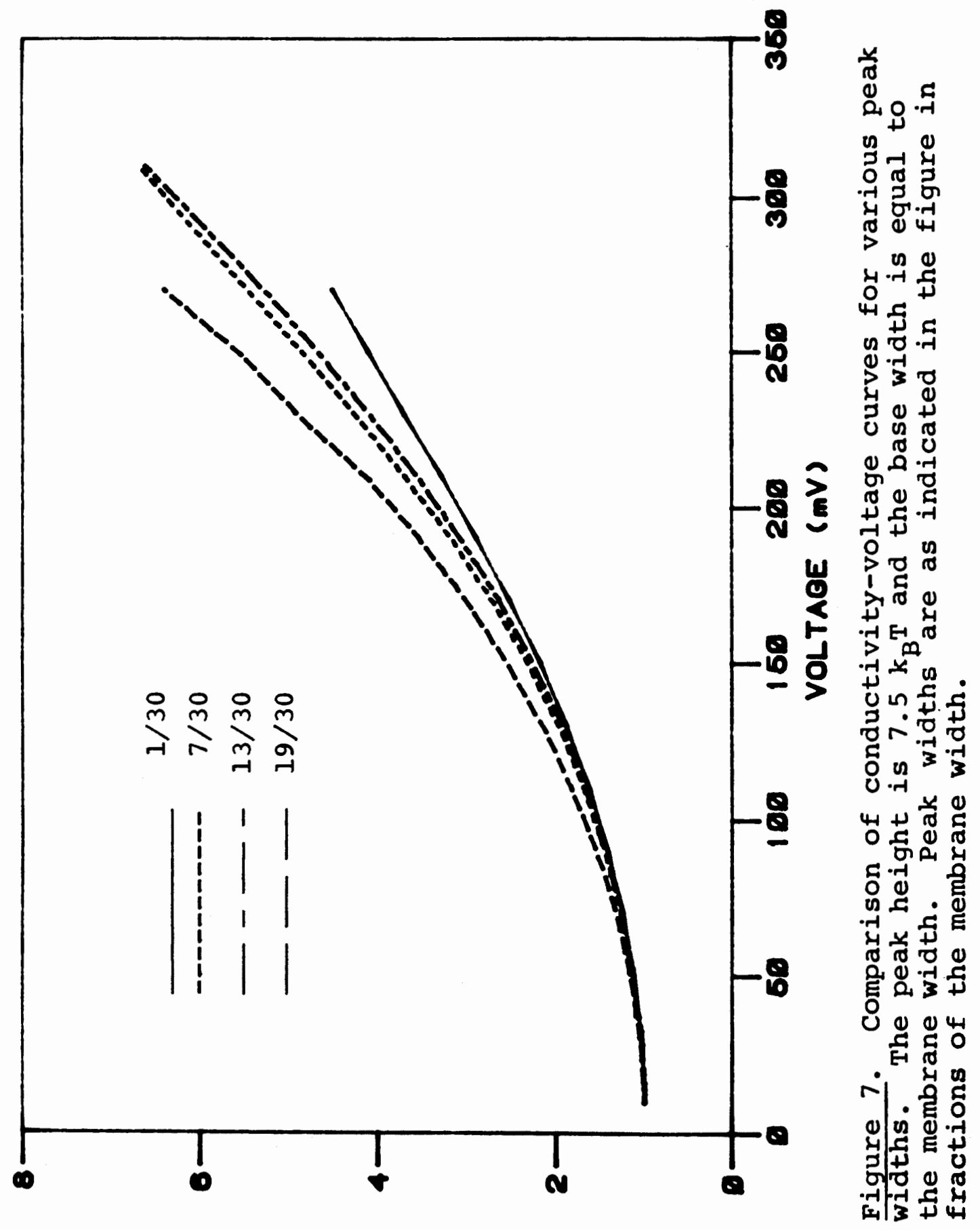




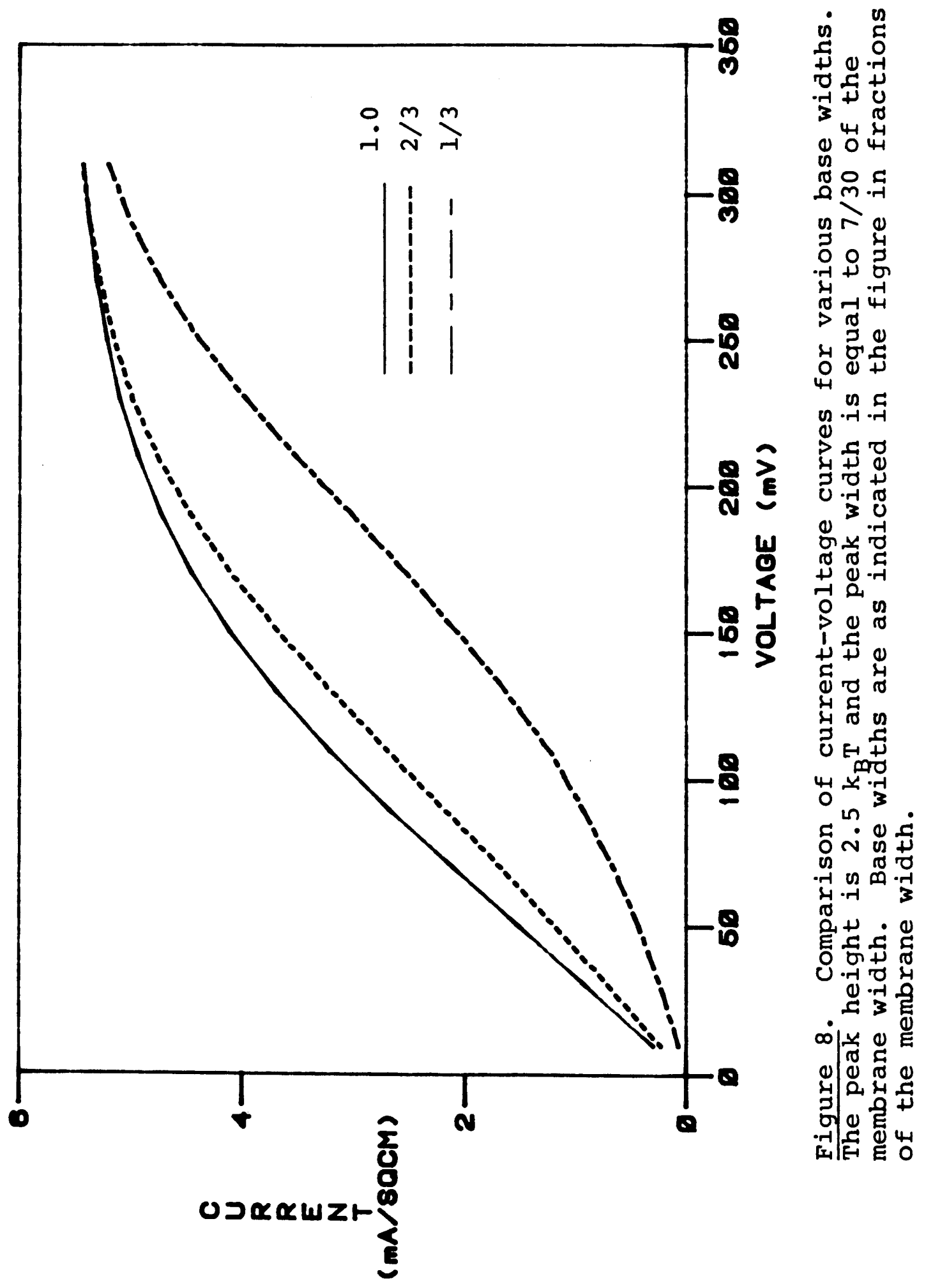




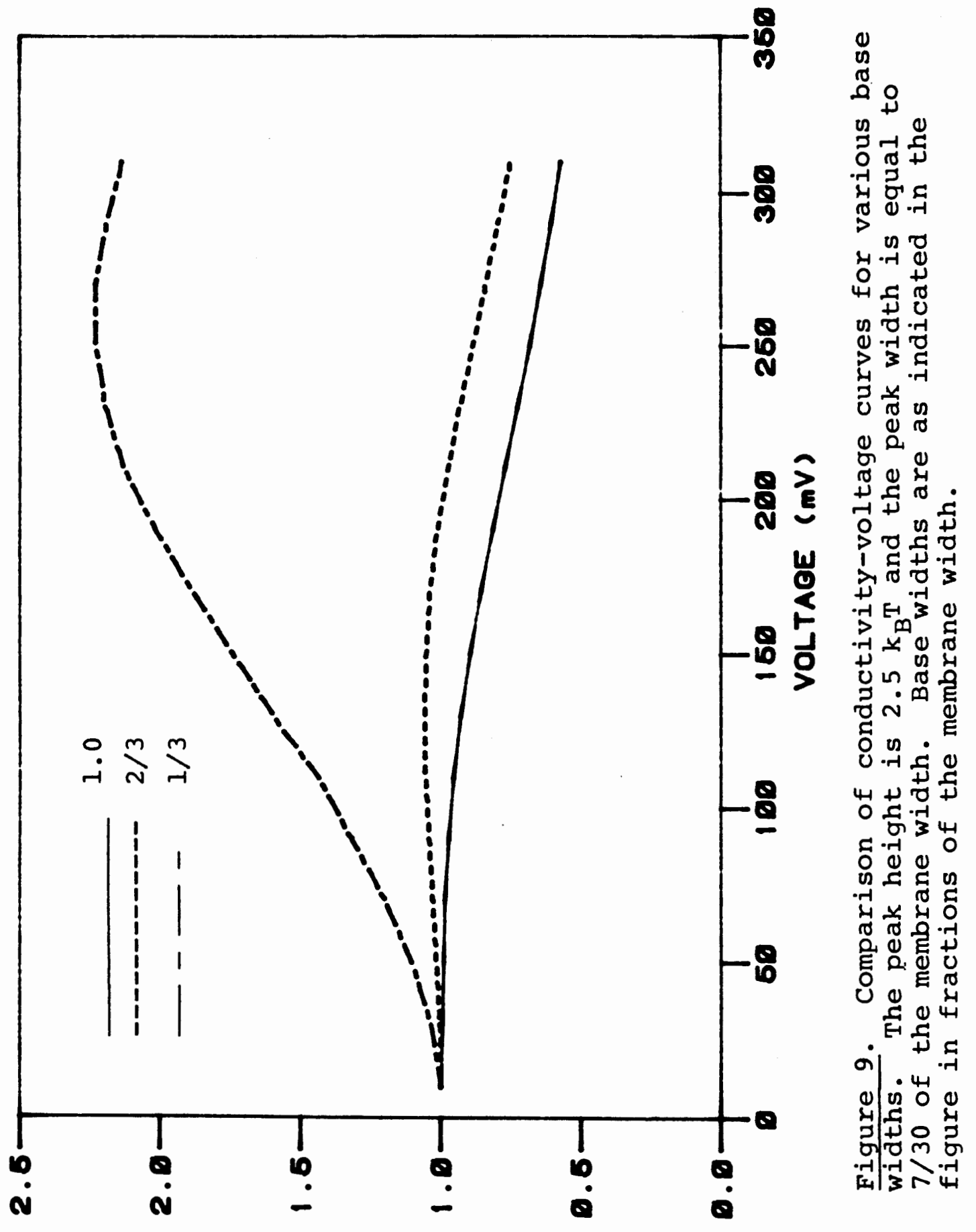




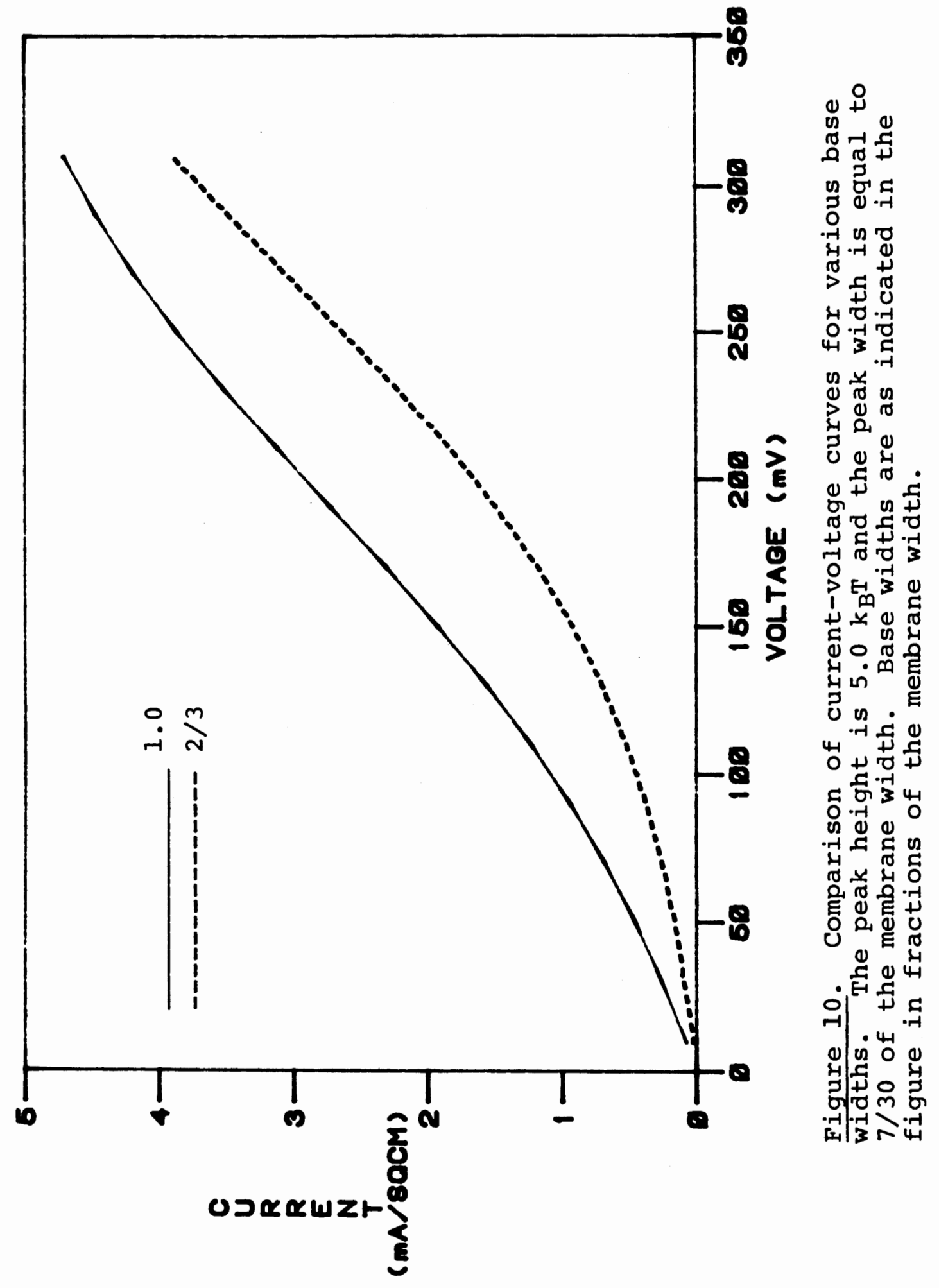




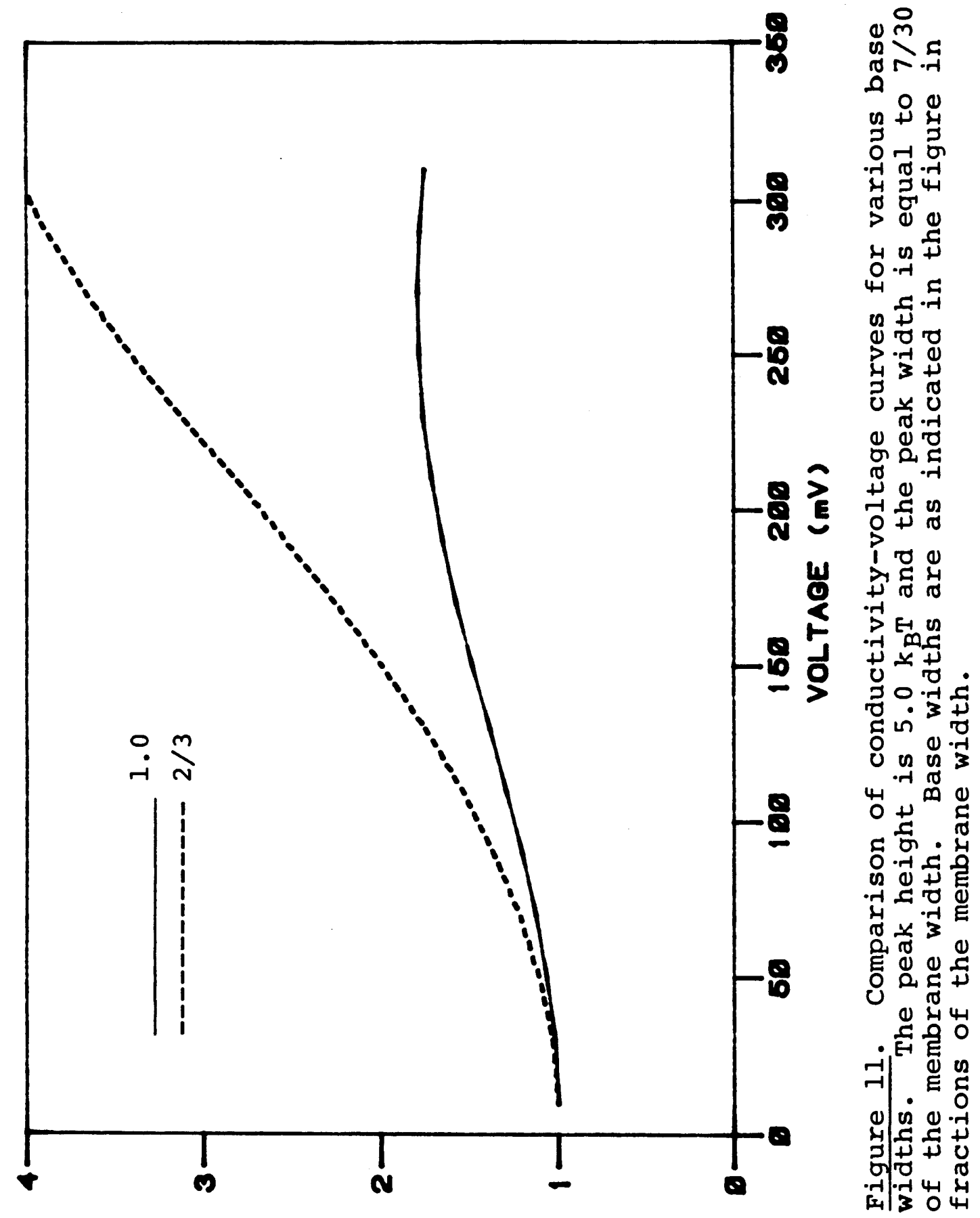


latter represents the same barrier as the long-dashed line in the former). ${ }^{2}$ Recalling the equations for interfacial transport, equations 17 and 18 ,

$$
j=\mp k\left(\sigma_{0}, d-F B c\right) \text {, }
$$

a possible means of explanation for this may be that the base width has a more pronounced effect upon the surface charge density (and also upon the total charge in the membrane) than does the peak width.

Total Charge in the Membrane

(1) When all other parameters are held constant, the total charge in the membrane, as determined in the numerical calculations, decreases with increasing base width, peak width, and peak height.

(2) The total charge also varies with applied voltage, decreasing dramatically for lower barriers, becoming more and more constant for higher barriers and wider peaks. Changing base widths does not give significant differences in this regard.

2 This is not the case in the constant field approximation. A comparison of the same two graphs, as well as others for this method of computation, shows that the degree of variation is comparable between peak and base width changes. 
(3) In comparing two different trapezoidal barriers, we very often find that the one which allows more charge to be in the membrane has a smaller current density for a given applied voltage. This is by no means an absolute rule, but it is, nonetheless, interesting when it occurs. Again, consideration of surface charge densities offers an explanation. For example, reducing the base width allows more charge into the membrane, but it is distributed more uniformly. The surface charge density at the interface is smaller, hence the current density is smaller also.

For physical systems in which the central barrier is not too high, a study of the variation of total charge with voltage may yield information as to the actual shape of the barrier. Unfortunately, in most lipid bilayer systems studied, the barrier is expected to be quite high and broad. Nevertheless, there are other reasons one may wish to measure the total charge in the membrane - e.g., to find the partition coefficient or to decide if the constant field approximation is appropriate - and in Appendix C, we suggest a possible method of measuring this. 


\section{CONSTANT FIELD VS. EXACT CALCULATIONS}

All trends noted in the previous section on the exact calculations, with the single exception as noted in footnote 2 , are observed in the constant field approximation as well.

While we must conclude that none of these effects are primarily due to ion-ion interactions, we also note that almost all of these trends are magnified by these interactions, as they are more pronounced in the exact calculations. The single exception to this statement is that the variation of total charge with voltage is greater in the constant field approximation. We also note here that the total charge in the membrane is always greater in the constant field approximation than in the exact calculations, sometimes by as much as 2 to 3 times at low voltages, this factor always decreasing with increasing voltage as the larger charge in the constant field approximation decreases more quickly.

For very high, broad barriers, the difference in total charge between the two calculations may become quite small, though this will not be the case if the base is narrow, and therefore flat near the interfaces where the charge density is highest. This is an indication of the importance of the 
actual shape of the barrier. For barriers with deep minima or flat regions near the interface, the absence of any barrier field in a region where the charge density is high will tend to increase the effect of any field due to the other ions in the membrane. As we see in Figures 12 and 13, the constant field approximation is much less adequate when there is a flat region in the barrier near the interface than when there is not, though in neither case is it a very good approximation. In the latter situation, the ion-barrier interactions overshadow the ionion interactions to some extent, while in the former case, the barrier actually acts to increase ion-ion interactions by forcing a large amount of charge to occupy a small region of space.

The consideration of ion-ion interactions is very important in the study of adsorption by bilayer membranes. In Figure 14, we plot calculated values of total charge at zero applied field against the aqueous ion concentration for three different barriers (compare Figures 1 and 2 of wang and Bruner (19)). At low concentrations, the relationship is linear, reflecting adherence to the constant field approximation (see Appendix A). As the charge density increases, so that ion-ion interactions become important, the relationship becomes nonlinear. The important thing to note is that the charge density at which this transition takes place is different for different barriers, indicating that the range 


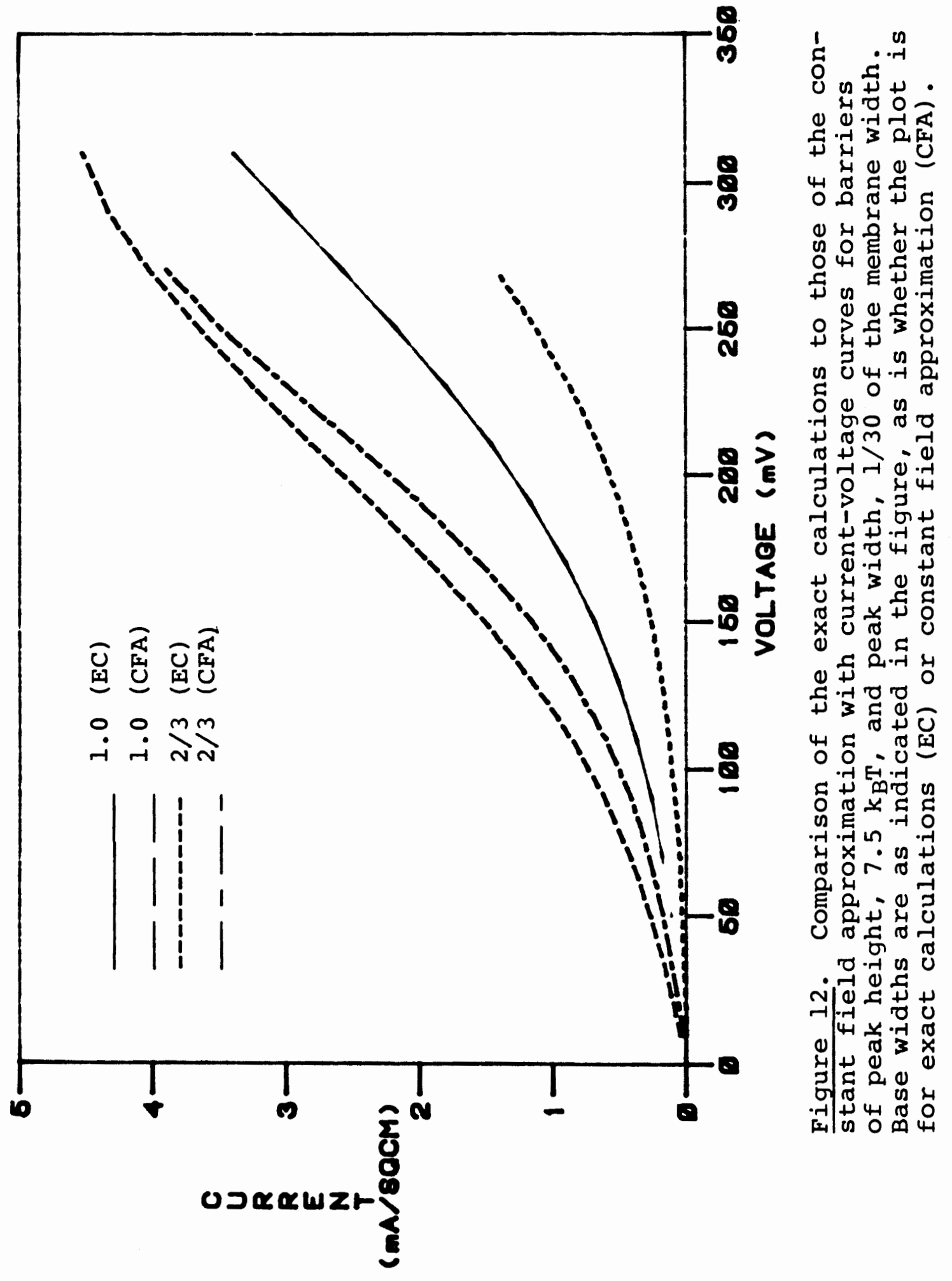




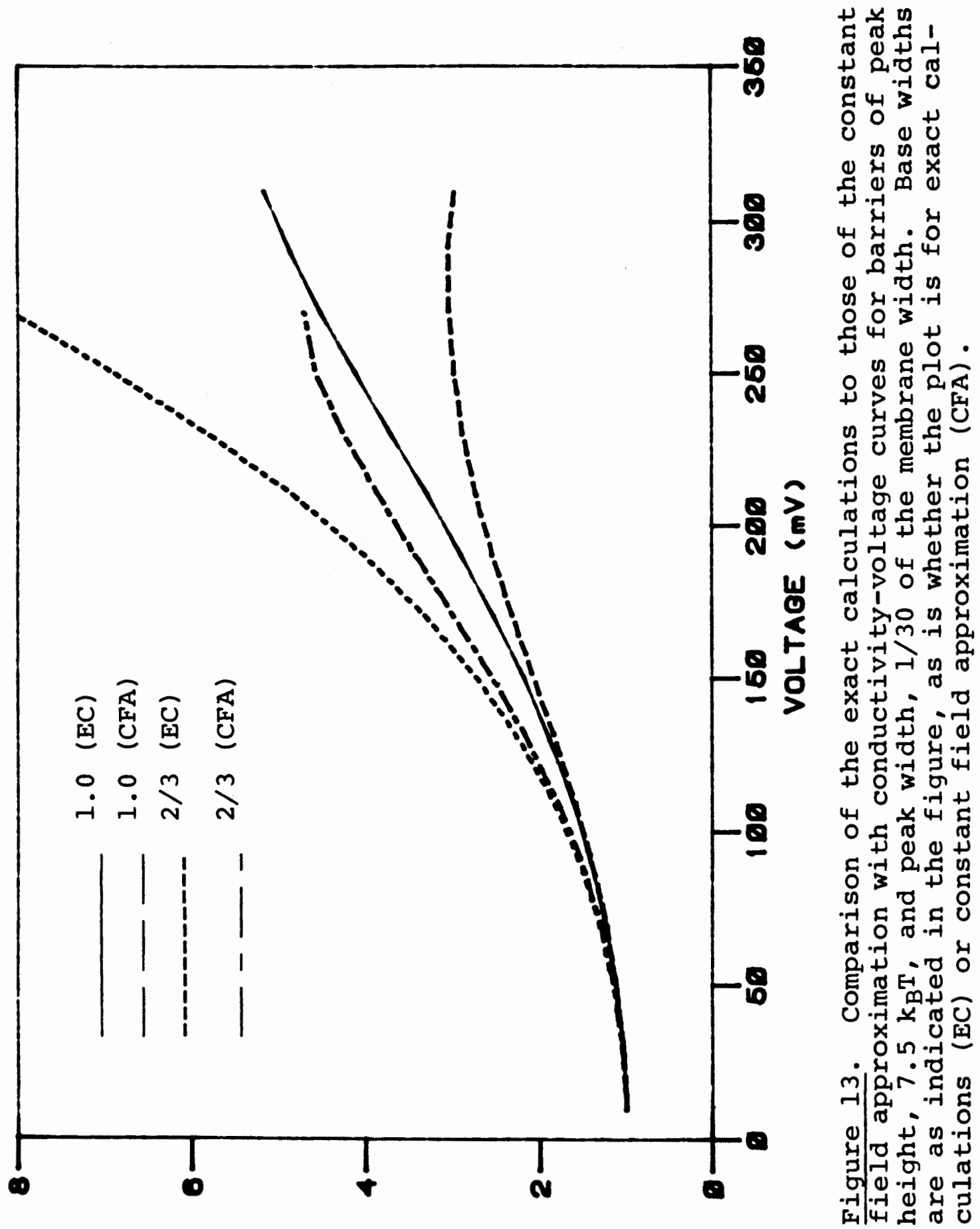




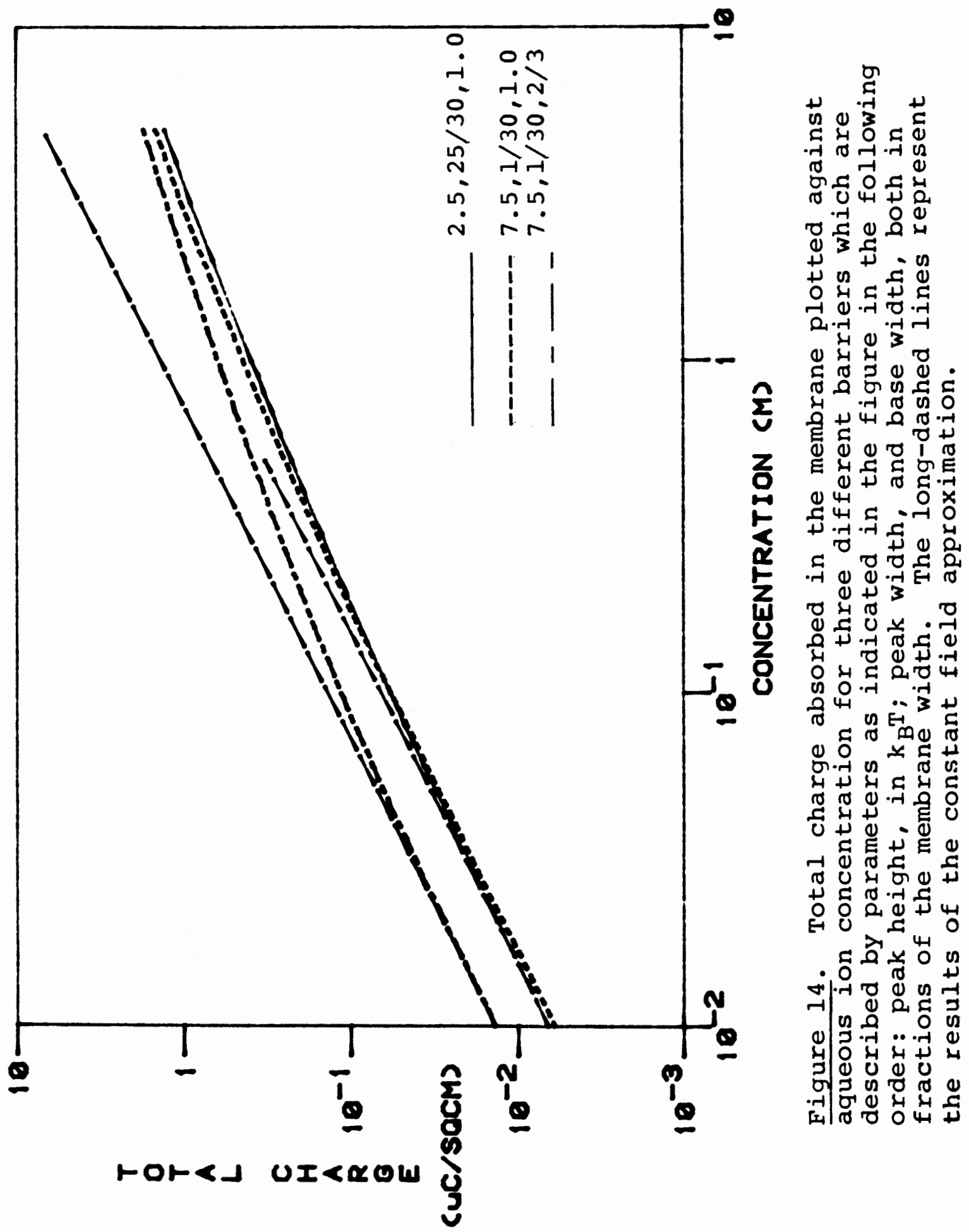


of validity of the constant field approximation - i.e., charge densities for which the relationship is linear will depend upon the shape of the barrier.

The differences between the exact calculations and the constant field approximation are striking at $c=1 \mathrm{M}$, not only in terms of the magnitude of the current density, which may differ by a factor of 3 to 4 , but also in terms of the shape of the $j-V$ relation. In some cases, the latter calculations predict a supralinear conductivity curve, while the former predicts a relation which is superlinear at low voltages, becoming supralinear only at quite high voltages (see Figure 15).

Superlinearity seems to indicate that the central barrier is rate-limiting (20). At high enough voltages, the interfacial processes will always become rate-limiting, but high barriers may be rate-limiting in a lower range of applied voltages. The fact that the exact calculations may give superlinearity for a given barrier while the constant field approximation does not, but never vice-versa, is an indication that ion-ion interactions tend to increase the importance of the central barrier in determining the transmembrane current. 


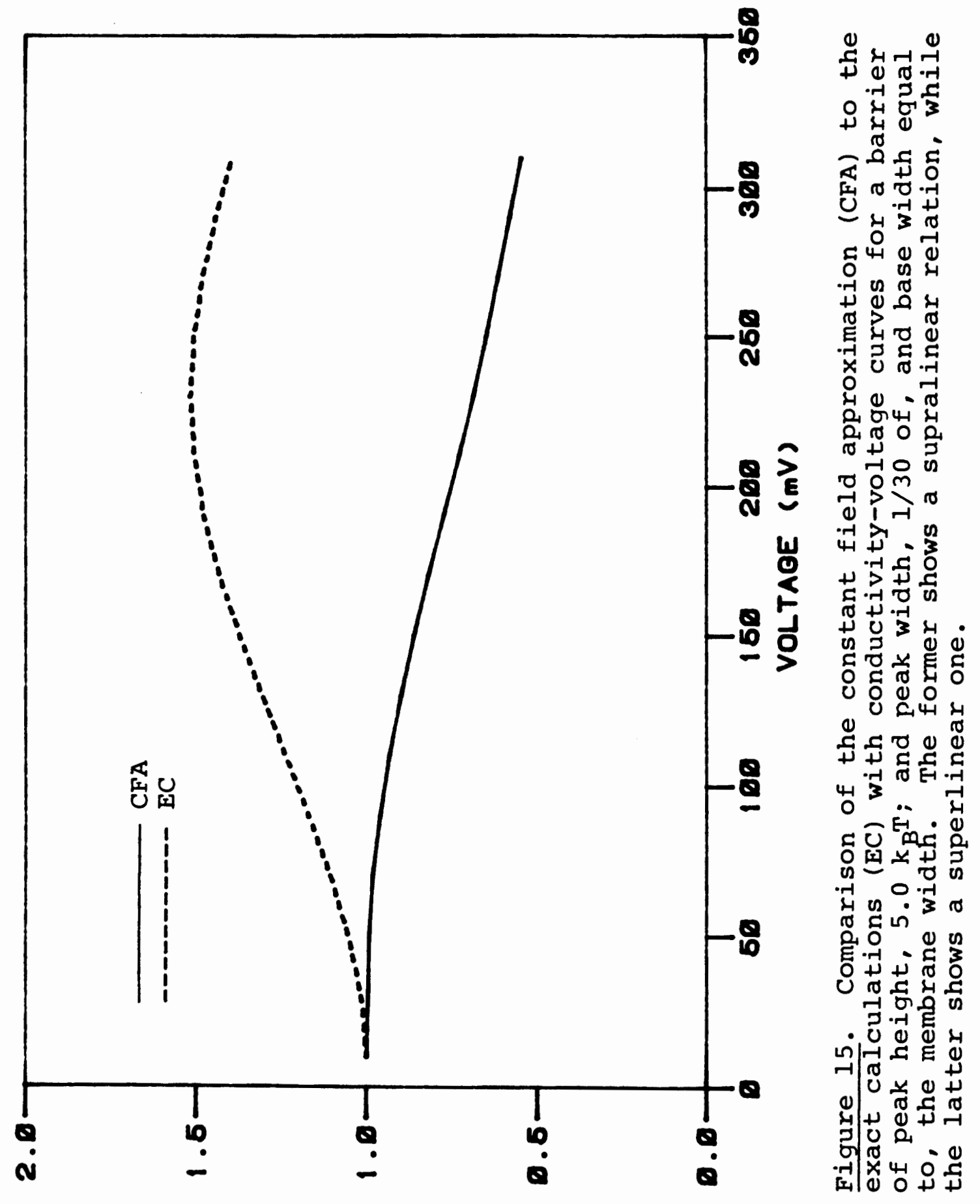


CHAPTER V

DISCUSSION

The results here presented indicate some points of interest on the application of electrodiffusion theory to ion transport across lipid bilayer membranes. In this section, we wish to conclude with a discussion of these results, comparing to experimental data where possible, and attempting to interpret them physically.

While the interfacial processes were not intended to be central to this paper, they clearly are important in placing certain limits upon the results. The most important of these is the appearance of a limiting current at high voltages. From equations 17 and 18 , it is easy to understand this limiting current in terms of a limiting surface charge density inside the membrane.

Combining these equations, we find

$$
\begin{aligned}
& j=k\left(\sigma_{o}-\sigma_{d}\right) / 2 \\
& \sigma_{o}+\sigma_{d}=2 F_{B C}
\end{aligned}
$$

The limiting current is seen to arise from one or the other of $\sigma_{0}$ and $\sigma_{\mathrm{d}}$ becoming negligible with respect to the re- 
maining one, and it is easy to see that this limiting current is given by

$$
j_{\text {lim }}=F B k C
$$

The presence of a limiting current depends upon the assumption that $B C$ is a constant, or at least does not increase indefinitely with increasing voltage. This assumption is quite appropriate to the actual physical systems, in which, if anything, $B c$ would be expected to decrease due to ion-ion interactions.

In attempting to interpret the results directly in terms of the potential energy barrier, it is worthwhile to consider the concept of the total potential energy of an ion in the membrane. This is essentially made up of three parts. Separate contributions come from interactions of the ion with the barrier, interactions of the ion with other ions present in the membrane, and from the applied potential.

As noted previously (and explained in terms of the interfacial processes), there are cases where one barrier will allow a smaller total charge in the membrane than another barrier, but a greater current density. This may be interpreted in terms of the central barrier in the following way: when the average potential energy of the barrier 
increases, ions tend to be driven from the membrane into the aqueous solution, where the potential energy is taken to be zero. However, the average potential energy of the ions remaining in the membrane increases, making it easier for them to surmount the barrier.

For example, the current increases with increasing base width, whereas the total charge decreases, while the current and total charge both decrease with increasing peak width. The increase of peak width does not in itself alter the average energy of the ions significantly due to the fact that the charge density is low in the region of the peak, but it does increase the region of high energy which the ions are required to traverse. When changing the base width, just the opposite is true. The shape of the barrier in the region of the peak is probably not as important as that near the interfaces in determining the current across actual membranes.

With wider peak widths, the increase of potential energy due to increased ion-ion interactions (the ions being confined to a smaller region) will oppose the effect of the increased region of high energy in decreasing the current, and this may explain why the current-voltage relation changes less with peak variation than with base variation, in which the direct effect, upon the current, of the change in barrier and the indirect effect through ion- 
ion interactions are in the same direction.

In some cases, it appears that ion-ion interactions may be overshadowed by the ion-barrier interactions. This does not occur when there is a flat region near the interface where the charge density is large. In this region, the barrier field vanishes, but the field due to charges will be very large. This may be important, especially in studying transport of hydrophobic ions for which the potential energy barrier has a deep minimum near the interface (21) .

The phenomenon of charge adsorption is also seen to be strongly dependent upon ion-ion interactions. For low charge densities, the contribution of the charge distributed through the volume of the membrane contributes significantly to the total charge in the membrane, but since the constant field approximation holds, the total charge is proportional to the aqueous concentration (see Appendix A). As the charge density increases, ion-ion effects become more important, and the contribution to the total charge from near the center of the membrane begins to fall below this linear relationship (see Figure 14 ). Most authors $(15,18)$ define the partition coefficient as the proportional constant between aqueous concentration and total numbers of ions per unit area in the membrane. The decrease in this proportional constant with increasing aqueous ion concentration 
has been observed experimentally $(14,15,19)$.

We have taken the values of our parameters from Stark, et. al. (17). Stark and Benz (9) have presented experimental data for the same system (valinomycin- $\mathrm{K}^{+}$in phosphatidyl inositol membranes). Our calculations fit this data quite closely for a few barriers which we have used, but the closest one is for a barrier of height 2.5 $k_{B} T_{i}$ peak width, $13 / 30$ and base width, 1.0 times the membrane thickness (see Figure 16).

We do not observe a transition from superlinearity to supralinearity with increasing aqueous concentration, as Stark and Benz (9) have reported. In fact, these calculations show the reverse trend (see Figure 17).

The inability of our calculations to compare favorably with this experimental result may be due to the simplifying assumptions which we have made. First of all, our choice of partition coefficient cannot be related to the experimental system. Secondly, the assumption that the uncomplexed carrier concentration is the same on both sides of the membrane may be quite unrealistic, and this may be able to explain the transition from super - to supra-linearity with increasing concentration. 


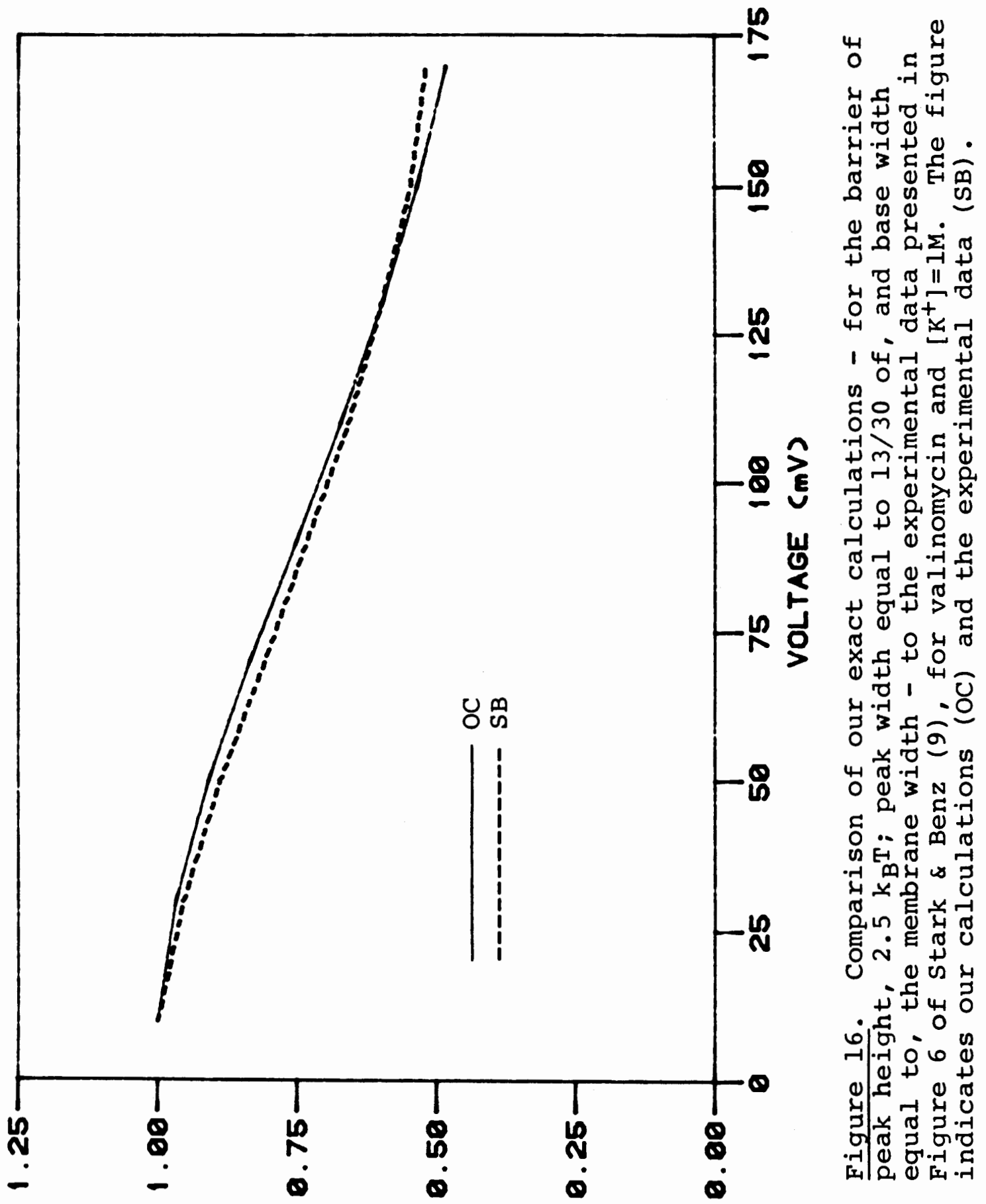




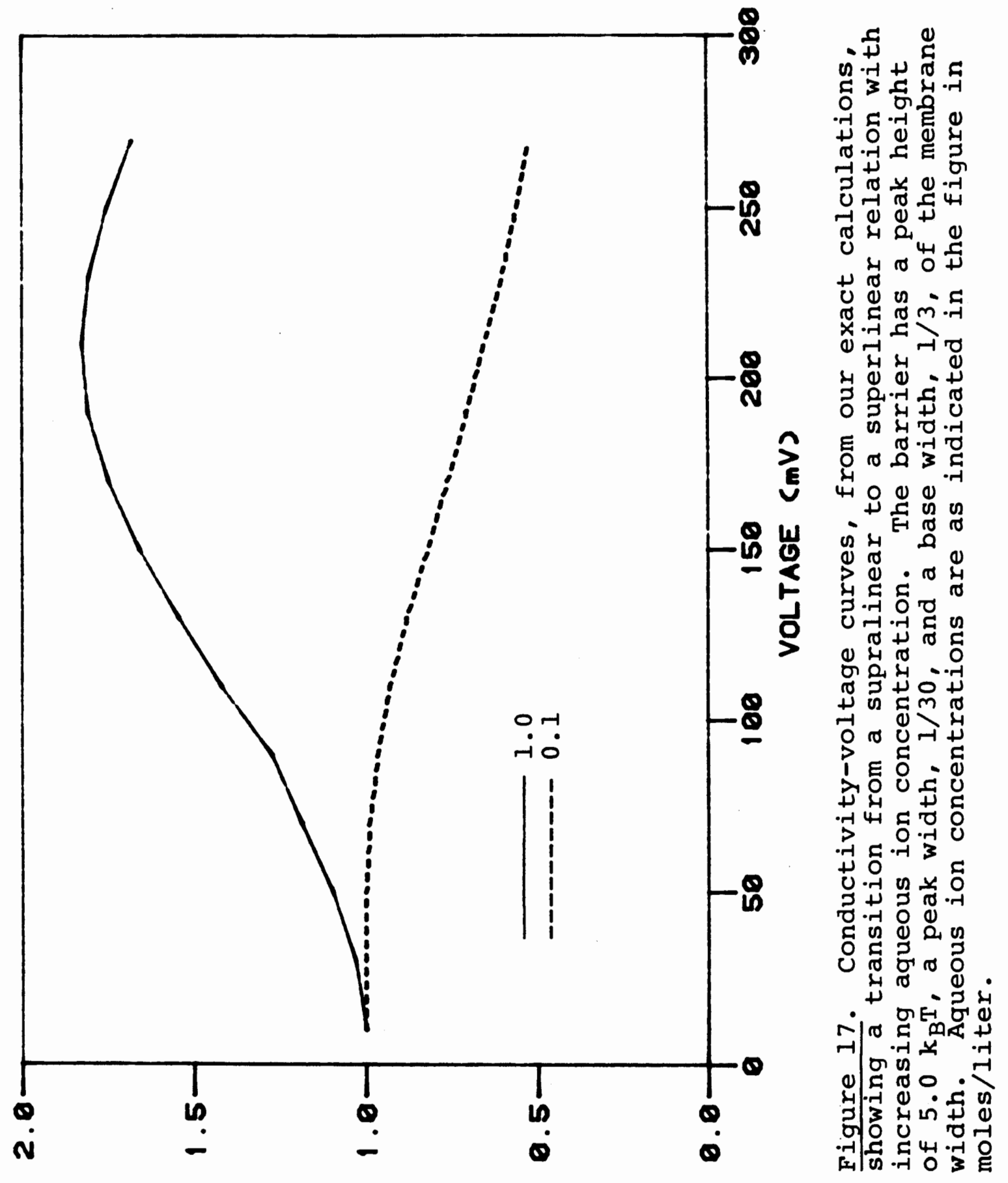




\section{REFERENCES}

1. Hladky, S.B. 1979. The Carrier Mechanism. Current Topics in Membranes and Transport 12 : 53-164.

2. Cohen, H. and J.W. Cooley 1965. The Numerical Solution of the Time-Dependent Nernst-Planck Equations. Biophysical Journal 5: 145-162.

3. De Levie, Robert and H. Moreira 1972. Transport of Ions of One Kind Through Thin Membranes. Journal of Membrane Biology 9: 241-260.

4. Leuchtag, H.R. and J.C. Swihert 1977. Steady-State Electrodiffusion. Biophysical Journal 17: 27-46.

5. Hays, Thomas R., Charles Q. Buckwalter, Jr., Sheng H. Lin, and Henry Eyring 1978. Ion Flow Through a Membrane: Concentration and Current Responses to a Step Potential Change. Proceedings of the National Academy of Sciences 75: 1612-1615.

6. Hall, James E., C.A. Mead, and Gabor Szabo 1973. A Barrier Model for Current Flow in Lipid Bilayer Membranes. Journal of Membrane Biology 11 : 75-97.

7. Attwell, David and Julian Jack 1978. The Interpretation of Membrane Current-Voltage Relations: A Nernst-Planck Analysis. Progress in Biophysics and Molecular Biology 34: 81-107.

8. Ginsburg, S. and D. Noble 1976. Use of CurrentVoltage Diagrams in Locating Peak Energy Barriers in Cell Membranes. Journal of Membrane Biology 29: 211-229.

9. Stark, G. and R. Benz 1971. The Transport of Potassium Through Lipid Bilayer Membranes by the Neutral Carriers Valinomycin and Monactin: Journal of Membrane Biology 5: 133-153.

10. Andersen, Olaf S. and Martin Fuchs 1975. Potential Energy Barriers to Ion Transport Within Lipid Bilayers. Biophysical Journal 15: 795-830. 
11. Neumcke, B. and P. Lăuger 1969. Nonlinear Electrical Effects in Lipid Bilayer Membranes. Biophysical Journal 9: 1160-1170.

12. Parsegian, A. 1969. Energy of an Ion Crossing a Low Dielectric Membrane: Solutions to Four Relevant Electrostatic Problems. Nature (London) 221 : $844-846$.

13. Neumcke, B. and P. Lauger 1970. Space Charge-Limited Conductance in Lipid Bilayer Membranes. Journal of Membrane Biology 3: 54-66.

14. Benz, R., P. Luluger and K. Janko 1976. Transport Kinetics of Hydrophobic Ions in Lipid Bilayer Membranes. Biochimica et Biophysica Acta 455: 701-720.

15. Ketterer, B., B. Neumcke, and P. Lduger 1971. Transport Mechanism of Hydrophobic Ions Through Lipid Bilayer Membranes. Journal of Membrane Biology 5: 225-245.

16. Offner, Franklin F. 1971. Nernst-Planck-Poisson Diffusion Equation:Numerical Solution of the Boundary Value Problem. Journal of Theoretical Biology 31: 215-227.

17. Stark, G., B. Ketterer, R. Benz, and P. Lauger 1971. The Rate Constants of Valinomycin-Mediated Ion Transport Through Thin Lipid Membranes. Biophysical Journal 11: 981-994.

18. Jordan, P.C. and G. Stark 1979. Kinetics of Transport of Hydrophobic Ions Through Lipid Membranes including Diffusion Polarization in the Aqueous Phase. Biophysical Chemistry 10: 273-287.

19. Wang, C.C. and L.J. Bruner 1978. Lipid-Dependent and Phloretin-Induced Modifications of Dipicrylamine Adsorption by Bilayer Membranes. Nature 272: 268-270.

20. Luuger, P. and G. Stark 1970. Kinetics of CarrierMediated Ion Transport Across Lipid Bilayer Membranes. Biochimica et Biophysica Acta 21l: 458-466. 
21. Lăuger, P., R. Benz, G. Stark, E. Bamberg, P.C. Jordan, A. Fahr, and W. Brock 1981. Relaxation Studies of Ion Transport Systems in Lipid Bilayer Membranes. Quarterly Reviews of Biophysics 14: 513-598. 


\section{APPENDIX A}

In the steady state, the current density is independent of the spatial coordinate and given by equation 6 :

$$
j=-D \frac{\rho(0)-\rho(d) e^{u}}{\int_{0} e^{-\frac{z F}{R T} \int_{0}^{x_{E}\left(x^{\wedge}\right) d x} d x}}
$$

or

$$
j=-D \frac{\rho(0)-\rho(x) e^{-\frac{z F}{R T} \delta_{0}^{x} E\left(x^{\prime}\right) d x^{\prime}}}{\delta_{0}^{x} e^{-\frac{z F}{R T} \int_{0}^{x^{\prime}} E\left(x^{\prime \prime}\right) d x^{\prime \prime}} d x^{\prime}}
$$

Defining

$$
\phi(x)=-\frac{z F}{R T} \int_{0}^{x} E\left(x^{\prime}\right) d x^{\prime},
$$

and rearranging equation $6 a$, we find the charge density given by

$$
\rho(x)=\left(\rho(0)-\sum_{D}^{j} f_{0}^{x} e^{\phi\left(x^{\prime}\right)} d x^{\prime}\right) e^{-\phi(x)}
$$

Using equation 2 , we have $\left(E_{v}(x)=E_{p}(x)-\frac{V}{d}\right)$

$E_{v}(x)=E_{v}(0)+\frac{4 \pi}{\varepsilon} \int_{0}^{x}\left(\rho(0)-\frac{j}{D} \int_{0}^{x^{\prime}} e^{\phi\left(x^{\prime \prime}\right)} d x^{\prime \prime}\right) e^{-\phi\left(x^{\prime}\right)} d x^{\prime}$.

We may integrate equation $A 2$ to find $Q$, the total charge in the membrane:

$Q=\rho(0) \int_{0}^{d} e^{-\phi(x)} d x-\frac{j}{D} \int_{0}^{d} e^{-\phi(x)} d x \int_{0}^{x_{e} \phi\left(x^{\prime}\right)} d x^{\prime}$

Integrating equation $A 3$ gives the applied voltage across the 
membrane

$$
\begin{aligned}
& V=-E_{v}(0) d-\frac{4 \pi}{\varepsilon} \rho(0) \int_{0} d x \int_{0}^{d} e^{-\phi\left(x^{\prime}\right)} d x^{\prime}+ \\
& \frac{4 \pi j}{\varepsilon D} \int_{0}^{d} d x \int_{0}^{x} e^{-\phi\left(x^{\prime}\right)} d x^{\prime} \int_{0}^{x^{\prime}} e^{\phi\left(x^{\prime \prime}\right)} d x^{\prime \prime}
\end{aligned}
$$

This last equation is interesting for the following reason: For the constant field approximation, and a given voltage we easily see that the current density is proportional to the charge density at the boundary. By equation A2, it follows that it is also proportional to $\rho(x)$ for arbitrary $x$, and also that $\rho(x)$ is proportional to $\rho(0)$. From equation $\mathrm{A} 4$, we reach the important conclusion that the current density is thus proportional to the total charge in the membrane. If we consider the boundary conditions,

$$
j=-k\left(\sigma_{0}-F_{\beta} C\right)
$$

and

$$
\sigma_{0}=\frac{h d}{2}\left(\rho(0)+\rho\left(\frac{d}{n}\right)\right),
$$

we see that $\sigma_{0}$ is proportional to the charge density at the boundary, which is proportional to the current density, which in turn, is proportional to the total charge. By the same argument, $\sigma_{\mathrm{d}}$ is proportional to the total charge. Finally, by equation 27 , the total charge in the membrane is proportional to the aqueous concentration of ions for these (and 
probably more general) boundary conditions in the constant field approximation. 


\section{APPENDIX B}

For carrier-mediated transport of ions, the steady state current across the interfaces is described by

$$
\begin{aligned}
& j=F\left(c k_{R} N_{s}^{\prime}-k N_{m s}^{\prime}\right) \\
& j=-F\left(c k_{R} N_{s}^{\prime \prime}-k N_{m s}^{\prime \prime}\right)
\end{aligned}
$$

where $k_{R}$ is the rate constant for the reaction between the carrier and the ion it will carry, $\mathrm{N}_{\mathrm{s}}^{\prime}\left(\mathrm{N}_{\mathrm{s}}^{\prime \prime}\right)$ and $\mathrm{N}_{\mathrm{ms}}^{\prime}\left(\mathrm{N}_{\mathrm{ms}}^{\prime \prime}\right)$ are the uncomplexed and complexed carrier concentrations, respectively, in the left (right) half of the membrane. These boundary conditions have the same form as the one's we have used, if $\mathrm{N}_{S}^{\prime}=\mathrm{N}_{S} "$ and is independent of voltage. The diffusion coefficient, $D$, is calculated from the relation (9) ( $\mathrm{k}_{\mathrm{ms}}$ ' the translocation rate constant)

$$
k_{m s}=2 D / d^{2}
$$

With $\mathrm{k}_{\mathrm{ms}}=1.82 \times 10^{4} \mathrm{sec}^{-1}$, we find

$$
D=1.46 \times 10^{-9} \mathrm{~cm}^{2} / \mathrm{sec} \text {. }
$$

We note here that we have chosen the value of $\beta$ somewhat arbitrarily, but to give charge densities in the membrane which are around .5 $\mu \mathrm{C} / \mathrm{cm}^{2}$ when $\mathrm{c}=1 \mathrm{M}$. 


\section{APPENDIX C}

The steady state current density at an applied voltage, $V_{0}$, is given by

$$
j_{s}\left(V_{0}\right)=-D\left(\frac{d \rho s}{d x}-\frac{z F}{R T}\left(E_{s}-\frac{V_{0}}{d}\right) \rho_{s}\right)
$$

Here, $E_{s}=E_{b}+E_{p}$, and the subscript, $s$, refers to the steady state values.

If we suddenly change the voltage from $v_{0}$ to $v_{l}$ and hold it constant, the current density immediately following the change is

$$
\begin{aligned}
j\left(V_{1}\right)_{t=0}=-D\left(\frac{\partial \rho_{s}}{\partial x}\right. & \left.-\frac{z F}{R T}\left(E_{s}-\frac{V_{0}}{d}+\frac{V_{0}-V_{1}}{d}\right)_{\rho_{s}}\right) \\
& +\frac{\varepsilon}{4 \pi} \frac{\partial E}{\partial t}
\end{aligned}
$$

We have assumed that the charging time of the external

circuit is fast enough that no charge redistribution has had time to take place within the membrane.

Subtracting equation $\mathrm{Cl}$ from equation $\mathrm{C} 2$ gives,

$$
\begin{aligned}
j\left(V_{1}\right)_{t=0}-j_{s}\left(V_{0}\right) & =\frac{z F D}{R T d}\left(V_{0}-V_{1}\right) \rho_{s}(x) \\
& +\frac{\varepsilon}{4 \pi} \underset{\frac{\partial E}{\partial t}}{ }
\end{aligned}
$$

If we now integrate across the membrane using 


$$
\int_{0}^{d} \frac{\partial E}{\partial t} d x=\frac{\partial}{\partial t} \int_{0}^{d} E d x=-\frac{\partial V_{1}}{\partial t}=0
$$

we find

$$
j\left(V_{1}\right)_{t=0}-j_{s}\left(V_{0}\right)=\frac{z F D}{R T d^{2}}\left(V_{0}-V_{1}\right) Q .
$$

By measuring the two current densities, we may calculate the total charge in the membrane if we know $D / d^{2}$. Note that by equation $\mathrm{B} 3$, this is related to the rate constant for translocation across the membrane. 